\title{
A kinetic approach to modeling general-texture evolution in two-dimensional polycrystalline grain growth
}

\author{
I. Yegorov ${ }^{\mathrm{a}, *}$, M. Emelianenko ${ }^{\mathrm{a}}$ \\ ${ }^{a}$ George Mason University, Department of Mathematical Sciences, 4400 University Drive, MS: 3F2, Exploratory Hall, \\ Fairfax, Virginia, USA, 22030
}

\begin{abstract}
Important statistical descriptors of grain boundary networks are misorientation distribution functions (often referred to as grain boundary character distributions), which show relative measures of interfaces with given misorientation parameters. This paper is aimed at extending the Boltzmann-type kinetic modeling framework developed in the previous work [1] to the two-dimensional case of general textures with no restrictions on possible grain orientations, but under the assumption that the grain boundary energy density depends only on the disorientation variable. In order to avoid an enormous computational complexity of treating the general-texture case, the kinetic model for polycrystalline grain growth is constructed from the very beginning in a specific discretized form with respect to the disorientation variable. A significant aspect of the developed approach is that its formulation requires a collection of a priori distributions which describe possible disorientations of grain boundaries connected in a triple junction. A separate numerical algorithm for approximating such distributions is proposed as applied to cubic crystal lattices. The numerical results obtained by the constructed model are in good agreement with the corresponding large-scale simulation results from the related literature.
\end{abstract}

Keywords: polycrystalline materials, grain growth, grain boundary, energy anisotropy, cubic lattice symmetry, disorientation, number-weighted disorientation distribution function, length-weighted disorientation distribution function, Boltzmann-type kinetic model.

\section{Introduction}

Many technologically useful materials such as metals and ceramics arise as polycrystalline microstructures. They are composed of single crystallites called grains. Interfaces between grains are called grain boundaries. The energetics and connectivity of grain boundary networks are expected to have a direct relationship to many macroscopic properties of materials across all scales of applications. In particular, these include functional properties such as electrical conductivity and lifetime properties like fracture toughness. Engineering of microstructures to achieve desired sets of performance characteristics is a major focus in materials science. An overview of grain boundary engineering was given in [2], and some related applications were discussed in [3-7].

Microstructures, i. e., grains' and grain boundaries' configurations, depend on many physical and chemical factors. In the current paper, the process of microstructural relaxation known as polycrystalline grain growth is considered. It is characterized by an increase in average size of grains at a high temperature after completion of recovery and recrystallization. In this situation, the further reduction in the interface energy can be reached only by decreasing the total measure of grain boundaries; see, for instance, $[8,9]$.

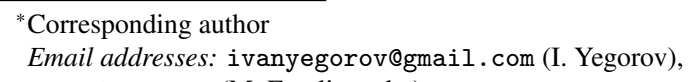

Important statistical descriptors of grain boundary networks are misorientation distribution functions (often referred to as grain boundary character distributions), which show relative measures of interfaces with given misorientation parameters; see [1, 9-24]. In order to investigate how the structure and time evolution of misorientation distribution functions depend on the grain boundary energy anisotropy and set of laws governing the dynamics at a microscopic scale, it is possible to conduct numerical experiments via well-known large-scale simulation approaches. In particular, these are Monte Carlo methods [1014], phase field models [12, 17, 18, 25], curvature-driven grain growth models [19-21], level set methods [23, 24], vertex models [26-28], etc. An alternative way is to develop kinetic models which are based on differential equations and, therefore, can be more computationally efficient, while focusing only on a restricted amount of essential characteristics.

Two novel frameworks for kinetic modeling of anisotropic grain growth with accompanying topological transitions were developed in [14, 22]. The paper [22] considered a simplified one-dimensional model and partially adopted a Boltzmann statistical mechanics perspective, which allowed to derive all-sufficient Boltzmann-type kinetic equations. The work [14] proposed a general partial differential equation, covering also two- and three-dimensional grain growth. This equation describes the change in numbers of grain boundaries with particular misorientation parameters as a result of topological transitions. However, all terms in its right-hand side are 
not known a priori, and, in [14], they were estimated by means of large-scale Monte Carlo simulations. Furthermore, in [14], specific boundary lengthening models were used to connect number-weighted misorientation distribution functions with area-weighted (in a three-dimensional case) or lengthweighted (in a two-dimensional case) ones.

The paper [1] modified and combined the two kinetic modeling frameworks from $[14,22]$ as applied to the particular case of polycrystalline thin films with so-called fiber textures. In this sufficiently narrow case, grains have nearly identical orientations in the axial direction perpendicular to a considered film, but random radial orientations in the plane of the film; see also [18]. In comparison with [14], the dependence of the kinetic model on large-scale simulations was essentially decreased. The developed approach has several shortcomings. However, for two nontrivial benchmark examples, its numerical results showed good agreement with the corresponding largescale simulation results from $[18,23]$. Also note that the constructed model is not exactly kinetic, because, instead of the time variable, it operates with the time-decreasing total number of grain boundaries.

The current work is aimed at extending the approach developed in [1] to the two-dimensional case of general textures with no restrictions on possible grain orientations, but under the assumption that the grain boundary energy density is a function of a single variable called disorientation. The disorientation of a grain boundary is defined as the smallest rotation angle from all the corresponding symmetrically equivalent misorientations. Although the misorientation of a grain boundary is characterized by three degrees of freedom, such reduction to disorientations is a commonly adopted and reasonable technique; see, for instance, $[10,13,14,23,24]$. Besides, the two additional variables specifying the orientation of a grain boundary plane are not included in our two-dimensional model, since they are related mainly to three-dimensional grain growth (where a full crystallographic characterization of a grain boundary requires not three but already five degrees of freedom); see [9]. Thus, disorientation distribution functions are the objects of the current investigations.

In order to avoid an enormous computational complexity of treating the general-texture case, the kinetic model is constructed from the very beginning in a specific discretized form with respect to the disorientation variable, as against the fibertexture considerations in [1].

Moreover, our attention is focused on the symmetry of cubic crystal lattices, because they take place in a wide class of technologically important metals and alloys; see, for instance, $[8,29]$. A significant aspect of the developed model is that its formulation requires a collection of a priori distributions which describe possible disorientations of grain boundaries connected in a triple junction. A separate numerical algorithm for approximating such distributions is also proposed in the current paper. The structure of the cubic disorientation domain in Rodrigues vector space (see [30,31]) is used in this algorithm.

The developed model is tested by comparing the numerical results, which it gives for several important benchmark examples, with the corresponding large-scale simulation results shown in $[14,23]$.

\section{Statement of the problem}

Consider two-dimensional grain growth in a polycrystalline thin film with a general texture. Each grain is characterized by a crystallographic orientation which is an orthogonal rotation matrix in the group $S O(3)$. All these orientations are determined in a single fixed three-dimensional Cartesian coordinate system (called sample or specimen coordinate system). We assume that, during growth or shrinkage, grains do not change their orientations.

Adopt the cubic lattice symmetry. Then, for any particular orientation $O$, there exist exactly 24 equivalent representations or, in other words, crystallographically related solutions. Each of them is generated by premultiplying the matrix $O$ by one of 24 specific matrices $S_{j}, j=\overline{1,24}$, called cubic symmetry operators:

$$
O_{j}=S_{j} \cdot O, \quad j=\overline{1,24} .
$$

Note that the inverse of a cubic symmetry operator and the product of two cubic symmetry operators are also cubic symmetry operators.

Let $O^{\prime}$ be one of the equivalent orientations for a grain $G^{\prime}$, and let $O^{\prime \prime}$ be that of another grain $G^{\prime \prime}$. Then the misorientation in the direction from $O^{\prime}$ to $O^{\prime \prime}$ has exactly $24^{2}=576$ equivalent representations

$$
M_{j k}^{O^{\prime}, O^{\prime \prime}}=S_{j} \cdot O^{\prime \prime} \cdot\left(O^{\prime}\right)^{-1} \cdot S_{k}, \quad j=\overline{1,24}, \quad k=\overline{1,24} .
$$

For every $j=\overline{1,24}, k=\overline{1,24}$, consider the axis/angle description corresponding to the matrix $M_{j k}^{O^{\prime}, O^{\prime \prime}}$ and having the nonnegative rotation angle

$$
\theta_{j k}^{O^{\prime}, O^{\prime \prime}}=\arccos \frac{\operatorname{tr} M_{j k}^{O^{\prime}, O^{\prime \prime}}-1}{2} \in[0, \pi]
$$

(the opposite angle together with the opposite direction of the rotation axis give an equivalent description). The disorientation angle or, in short, disorientation between $O^{\prime}, O^{\prime \prime}$ is defined as the smallest nonnegative rotation angle

$$
\theta^{O^{\prime \prime}, O^{\prime}}=\theta^{O^{\prime}, O^{\prime \prime}} \stackrel{\text { def }}{=} \min _{j=\overline{1,24}, k=\overline{1,24}} \theta_{j k}^{O^{\prime}, O^{\prime \prime}}=\min _{j=\overline{1,24}, k=\overline{1,24}} \theta_{j k}^{O^{\prime \prime}, O^{\prime}} .
$$

If $G^{\prime}$ and $G^{\prime \prime}$ are neighboring grains, then the disorientation between $G^{\prime}, G^{\prime \prime}$ is interpreted as the disorientation between $O^{\prime}, O^{\prime \prime}$.

The disorientation angle for an arbitrary rotation matrix $R \in$ $S O(3)$ can be determined as

$$
\begin{aligned}
\theta^{R} & \stackrel{\text { def }}{=} \underset{j=\frac{\min }{1,24}, k=\overline{1,24}}{ } \alpha^{S_{j} \cdot R \cdot S_{k}}= \\
& =\min _{j=1,24} \alpha^{S_{j} \cdot R}=\min _{k=1,24} \alpha^{R \cdot S_{k}},
\end{aligned}
$$

where the notation

$$
\alpha \stackrel{\text { def }}{=} \arccos \frac{\operatorname{tr} Q-1}{2}
$$


is used for the nonnegative rotation angle in the corresponding axis/angle description of a rotation matrix $Q \in S O(3)$. Indeed, for every $k=\overline{1,24}, j=\overline{1,24}$, there exists $l_{k j} \in\{1,2, \ldots, 24\}$ such that $S_{k} \cdot S_{j}=S_{l_{k j}}$ and, hence,

$$
\begin{aligned}
\operatorname{tr}\left(S_{j} \cdot R \cdot S_{k}\right) & =\operatorname{tr}\left(S_{k} \cdot\left(S_{j} \cdot R \cdot S_{k}\right) \cdot S_{k}^{-1}\right)= \\
= & \operatorname{tr}\left(S_{k} \cdot S_{j} \cdot R\right)=\operatorname{tr}\left(S_{l_{k j}} \cdot R\right), \\
\operatorname{tr}\left(S_{j} \cdot R \cdot S_{k}\right) & =\operatorname{tr}\left(S_{j}^{-1} \cdot\left(S_{j} \cdot R \cdot S_{k}\right) \cdot S_{j}\right)= \\
= & \operatorname{tr}\left(R \cdot S_{k} \cdot S_{j}\right)=\operatorname{tr}\left(R \cdot S_{l_{k j}}\right) .
\end{aligned}
$$

This implies (5). In particular, we can simplify (4) as

$$
\theta^{O^{\prime \prime}, O^{\prime}}=\theta^{O^{\prime}, O^{\prime \prime}}=\min _{j=1,24} \alpha^{S_{j} \cdot O^{\prime \prime} \cdot\left(O^{\prime}\right)^{-1}}=\min _{k=1,24} \alpha^{O^{\prime \prime} \cdot\left(O^{\prime}\right)^{-1} \cdot S_{k}}
$$

For the cubic lattice symmetry, the set of possible disorientations is the line segment $\left[0, \theta_{\max }\right]$ with

$$
\theta_{\max } \approx 62.8 \cdot \frac{\pi}{180}=62.8^{\circ}
$$

A more detailed introduction to the mentioned concepts and their fundamental properties can be found, for instance, in [2931].

Assume that

$$
\gamma:\left[0, \theta_{\max }\right] \rightarrow[0,+\infty)
$$

is the grain boundary energy density depending only on the disorientation variable, $t \in[0, T]$ is the time variable, and the total number and length of grain boundaries in the considered twodimensional system are $N(t)$ and $L(t)$, respectively. For every $t \in[0, T]$, introduce the measure densities $N(\cdot, t)$ and $L(\cdot, t)$ supported on the line segment $\left[0, \theta_{\max }\right]$ and such that the number and total length of boundaries with disorientations from a Borel set $B \subseteq\left[0, \theta_{\max }\right]$ at time $t$ are the integrals $\int_{B} N(\theta, t) d \theta$ and $\int_{B} L(\theta, t) d \theta$, respectively. In particular,

$$
\begin{array}{r}
\int_{0}^{\theta_{\max }} N(\theta, t) d \theta=N(t), \int_{0}^{\theta_{\max }} L(\theta, t) d \theta=L(t) \\
\forall t \in[0, T] .
\end{array}
$$

Also let $\bar{L}(t)$ be the average boundary length in the whole system, and let $\bar{L}(\theta, t)$ be the average length for a particular disorientation $\theta \in\left[0, \theta_{\max }\right]$ :

$$
\begin{aligned}
\bar{L}(t) \stackrel{\text { def }}{=} & \frac{L(t)}{N(t)}, \quad \bar{L}(\theta, t) \stackrel{\text { def }}{=} \frac{L(\theta, t)}{N(\theta, t)} \\
& \forall \theta \in\left[0, \theta_{\max }\right] \quad \forall t \in[0, T] .
\end{aligned}
$$

Now, according to $[1,14,18]$, introduce the number- and length-weighted disorientation distribution functions (DDFs):

$$
\begin{aligned}
\rho_{n}(\theta, t) \stackrel{\text { def }}{=} & \frac{N(\theta, t)}{N(t)}, \quad \rho_{l}(\theta, t) \stackrel{\text { def }}{=} \frac{L(\theta, t)}{L(t)} \\
& \forall \theta \in\left[0, \theta_{\max }\right] \quad \forall t \in[0, T] .
\end{aligned}
$$

The length-weighted DDF $\rho_{l}$ can be referred to as grain boundary character distribution (GBCD); see, for instance, [20]. As in $[1,14]$, from $(11),(12)$, we obtain

$$
\frac{\rho_{l}(\theta, t)}{\rho_{n}(\theta, t)}=\frac{\bar{L}(\theta, t)}{\bar{L}(t)} \quad \forall \theta \in\left[0, \theta_{\max }\right] \quad \forall t \in[0, T] .
$$

As in [1], we do not include such characteristic as the grain boundary mobility (discussed, for instance, in $[12,17]$ ) into the constructed model, i. e., mobility isotropy is considered. This assumption is reasonable, since, according to the results of Monte Carlo and phase field simulations of two- and threedimensional grain growth presented in [12, 13, 17], misorientation (or disorientation) distribution functions resulting from mobility anisotropy subject to energy isotropy are negligibly different from those developed with both mobility and energy isotropy.

We describe topological transitions accompanying twodimensional grain growth as either neighbor switchings or grain disappearances; see, for instance, [14, 27, 28]. Suppose that initial microstructures do not contain two-sided grains and only three-sided grains are allowed to disappear. Then, in particular, transformations of three-sided grains into two-sided grains by neighbor switchings are forbidden, and, therefore, two-sided grains cannot appear. In [1, section 2], some arguments in favor of this assumption are given.

Let the functions $N_{s}(t)$ and $N_{d}(t)$ represent total numbers of neighbor switchings and grain disappearances occuring up to a varying time moment $t\left(N_{s}(0)=N_{d}(0)=0\right)$. As in [1], we also impose the idealizing assumption that $N(\theta, t), N(t), N_{s}(t), N_{d}(t)$ change with time not discretely but continuously.

The main goal of this paper is to develop a method to estimate the number- and length-weighted DDFs (12) for the adopted general-texture case and cubic lattice symmetry. With the help of (13), we reduce the problem of constructing both of them to the problem of constructing $\rho_{n}$ and the right-hand side of (13); see also $[1,14]$. We approximate the number-weighted DDF via a kinetic model extending the fiber-texture approach of [1] and estimate the quotient (13) by means of additional framework.

\section{Discretization with respect to the disorientation variable}

In order to decrease the computational complexity of the kinetic model for general textures, it is reasonable to discretize the set of possible disorientations from the very beginning.

Take a sufficiently dense grid

$$
0=\theta_{0}<\theta_{1}<\theta_{2}<\ldots<\theta_{r-1}<\theta_{r}=\tilde{\theta}_{\max }
$$

on the interval $\left[0, \tilde{\theta}_{\max }\right]$ for some constant $\tilde{\theta}_{\max }$ such that

$$
\theta_{r-1}<\theta_{\max }<\tilde{\theta}_{\max }
$$

Extend the function (9) from $\left[0, \theta_{\max }\right]$ to $\left[0, \tilde{\theta}_{\max }\right]$ merely by

$$
\gamma(\theta) \stackrel{\text { def }}{=} \gamma\left(\theta_{\max }\right) \quad \forall \theta \in\left(\theta_{\max }, \tilde{\theta}_{\max }\right] .
$$


For every $i=\overline{1, r}$, consider all disorientations from the subinterval $\left[\theta_{i-1}, \theta_{i}\right)$ together, and let the value

$$
\gamma_{i} \stackrel{\text { def }}{=} \gamma\left(\theta_{i}^{\text {mid }}\right)
$$

of the grain boundary energy density at the midpoint

$$
\theta_{i}^{\text {mid }} \stackrel{\text { def }}{=} \frac{\theta_{i-1}+\theta_{i}}{2}
$$

represent all its values on $\left[\theta_{i-1}, \theta_{i}\right)$. Furthermore, for any $t \in$ $[0, T]$ and $i=\overline{1, r}$, let the value $\bar{L}\left(\theta_{i}^{\mathrm{mid}}, t\right) / \bar{L}(t)$ of the quotient (13) at the point $\left(\theta_{i}^{\mathrm{mid}}, t\right)$ represent its values at points $(\theta, t)$ for all $\theta \in\left[\theta_{i-1}, \theta_{i}\right)$.

Then we can approximate the number- and length-weighted DDFs by the vector-functions $\left\{\rho_{n}^{i}(t)\right\}_{i=1}^{r}$ and $\left\{\rho_{l}^{i}(t)\right\}_{i=1}^{r}$, respectively, where

$$
\begin{aligned}
& \rho_{n}^{i}(t) \stackrel{\operatorname{def}}{=} \int_{\theta_{i-1}}^{\theta_{i}} \rho_{n}(\theta, t) d \theta, \\
& \rho_{l}^{i}(t) \stackrel{\text { def }}{=} \frac{\frac{\bar{L}\left(\theta_{i}^{\text {mid }}, t\right)}{\bar{L}(t)} \cdot \rho_{n}^{i}(t)}{\sum_{j=1}^{r} \frac{\bar{L}\left(\theta_{j}^{\text {mid }}, t\right)}{\bar{L}(t)} \cdot \rho_{n}^{j}(t)} \\
& \forall t \in[0, T], \quad i=\overline{1, r} .
\end{aligned}
$$

It is obvious that

$$
\sum_{i=1}^{r} \rho_{n}^{i}(t)=\sum_{i=1}^{r} \rho_{l}^{i}(t)=1 \quad \forall t \in[0, T] .
$$

Also denote

$$
N^{i}(t) \stackrel{\text { def }}{=} \int_{\theta_{i-1}}^{\theta_{i}} N(\theta, t) d \theta \quad \forall t \in[0, T], \quad i=\overline{1, r} .
$$

Due to (12),(19), we have

$$
N^{i}(t)=N(t) \cdot \rho_{n}^{i}(t) \quad \forall t \in[0, T], \quad i=\overline{1, r} .
$$

\section{System of kinetic equations for the number- weighted DDF}

As in [1], consider four types of possible critical events:

- creation of a grain boundary with a particular disorientation as a result of a neighbor switching;

- removal of a grain boundary with a particular disorientation as a result of a neighbor switching;

- removal of a zero-length grain boundary with a particular disorientation as a result of a grain disappearance;

- removal of a nonzero-length grain boundary with a particular disorientation as a result of a grain disappearance.

For every $t \in[0, T]$ and $i=\overline{1, r}$, adopt the following notations:
- $P_{s,+}^{i}(t)$ is the probability of lying in the interval $\left[\theta_{i-1}, \theta_{i}\right)$ for the disorientation of a grain boundary created as a result of a neighbor switching at the moment $t$;

- $P_{s,-}^{i}(t)$ is the probability of lying in the interval $\left[\theta_{i-1}, \theta_{i}\right)$ for the disorientation of a grain boundary removed as a result of a neighbor switching at the moment $t$;

- $P_{d, 1}^{i}(t)$ is the probability of lying in the interval $\left[\theta_{i-1}, \theta_{i}\right)$ for the disorientation of a grain boundary removed with zero length as a result of a grain disappearance at the moment $t$;

- $P_{d, 2}^{i}(t)$ is the probability of lying in the interval $\left[\theta_{i-1}, \theta_{i}\right)$ for the disorientation of a grain boundary removed with nonzero length as a result of a grain disappearance at the moment $t$.

One can easily see that

$$
\begin{aligned}
\sum_{i=1}^{r} P_{s,+}^{i}(t) & =\sum_{i=1}^{r} P_{s,-}^{i}(t)=\sum_{i=1}^{r} P_{d, 1}^{i}(t)= \\
& =\sum_{i=1}^{r} P_{d, 2}^{i}(t)=1 \quad \forall t \in[0, T] .
\end{aligned}
$$

In section 6 , the introduced probabilities will be expressed in terms of the sought-for number-weighted DDF and some auxiliary a priori distributions discussed in section 5 .

Also let $\lambda(t)$ be the ratio of the neighbor switchings' rate to the grain disappearances' rate at the moment $t$.

Similarly to [1, section 3$]$, by starting with the basic relation (an analog of [1, formula (8)])

$$
\begin{aligned}
N^{i}(t+ & \Delta t)-N^{i}(t)= \\
= & \left(P_{s,+}^{i}(t)-P_{s,-}^{i}(t)\right) \cdot\left(N_{s}(t+\Delta t)-N_{s}(t)\right)- \\
& -P_{d, 1}^{i}(t) \cdot\left(N_{d}(t+\Delta t)-N_{d}(t)\right)- \\
& -P_{d, 2}^{i}(t) \cdot 2\left(N_{d}(t+\Delta t)-N_{d}(t)\right),
\end{aligned}
$$

we can derive the following system of ordinary differential equations for the number-weighted DDF (an analog of [1, formula (11)]:

$$
\begin{aligned}
\rho_{n}^{i}(N) & +N \cdot \frac{d \rho_{n}^{i}(N)}{d N}= \\
= & -\frac{\lambda(N)}{3} \cdot\left(P_{s,+}^{i}(N)-P_{s,-}^{i}(N)\right)+ \\
& +\frac{1}{3} \cdot P_{d, 1}^{i}(N)+\frac{2}{3} \cdot P_{d, 2}^{i}(N) \\
& \forall N \in\left[N_{\min }, N_{0}\right], \quad i=\overline{1, r} .
\end{aligned}
$$

Here

$$
\left.N_{0} \stackrel{\text { def }}{=} N\right|_{t=0},\left.\quad N_{\text {min }} \stackrel{\text { def }}{=} N\right|_{t=T}
$$

are parameters. The initial conditions are taken as

$$
\rho_{n}^{i}\left(N_{0}\right)=\rho_{n, 0}^{i}, \quad i=\overline{1, r}
$$

The vector $\left\{\rho_{n, 0}^{i}\right\}_{i=1}^{r}$ is a discretized representation of the initial number-weighted DDF. When deriving (25), it is assumed 
that, when a three-sided grain disappears, only one of its sides is eliminated with zero length. We also consider $N=N(t)$ as a strictly decreasing real-valued function and replace the dependence on $t$ with the dependence on $N$. Let us write a simple numerical scheme for Cauchy problem (25),(27):

$$
\left\{\begin{aligned}
& \rho_{n}^{i}(N-\Delta N)=\rho_{n}^{i}(N)+\frac{\Delta N}{N} \cdot\left(\rho_{n}^{i}(N)+\right. \\
&+\frac{\lambda(N)}{3} \cdot\left(P_{s,+}^{i}(N)-P_{s,-}^{i}(N)\right)- \\
&\left.-\frac{1}{3} \cdot P_{d, 1}^{i}(N)-\frac{2}{3} \cdot P_{d, 2}^{i}(N)\right), \\
& i=\overline{1, r}, \\
& \Delta N>0 \quad \text { is sufficiently small, } \\
& \rho_{n}^{i}\left(N_{0}\right)=\rho_{n, 0}^{i}, \quad i=\overline{1, r} .
\end{aligned}\right.
$$

\section{Auxiliary a priori disorientation distributions}

In comparison with the fiber-texture case treated in [1], a substantial difficulty of treating the general-texture case is that some additional quantities are required in order to represent $P_{s,+}^{i}, P_{s,-}^{i}, P_{d, 1}^{i}, P_{d, 2}^{i}, i=\overline{1, r}$, via $\left\{\rho_{n}^{j}\right\}_{j=1}^{r}$. They should describe a priori possible disorientations for a grain boundary which is connected in a triple junction together with two other boundaries having given disorientations.

Let us propose a numerical algorithm to construct the needed quantities as discrete distributions. Namely, for every $i=\overline{1, r}$, $i_{1}=\overline{1, r}, i_{2}=\overline{1, r}$, let $\eta_{i_{1}, i_{2}}^{i}$ be the probability of lying in the interval $\left[\theta_{i-1}, \theta_{i}\right)$ for the disorientation of a grain boundary which is connected in a triple junction with two other boundaries having disorientations from the intervals $\left[\theta_{i_{1}-1}, \theta_{i_{1}}\right)$ and $\left[\theta_{i_{2}-1}, \theta_{i_{2}}\right)$. It is obvious that

$$
\begin{gathered}
\eta_{i_{1}, i_{2}}^{i}=\eta_{i_{2}, i_{1}}^{i}, \quad i=\overline{1, r}, \quad i_{1}=\overline{1, r}, \quad i_{2}=\overline{1, r}, \\
\sum_{i=1}^{r} \eta_{i_{1}, i_{2}}^{i}=1, \quad i_{1}=\overline{1, r}, \quad i_{2}=\overline{1, r} .
\end{gathered}
$$

Here it is convenient to use such alternative descriptors of rotation matrices in $S O(3)$ as unit quaternions and Rodrigues vectors; a very brief introduction to them is given, for instance, in [31].

There is a two-to-one homomorphism between the group of unit quaternions and $S O(3)$. For every $j=\overline{1,24}$, let $s_{j} \stackrel{\text { def }}{=}$ $\left(s_{j}^{0}, s_{j}^{1}, s_{j}^{2}, s_{j}^{3}\right)$ be a unit quaternion corresponding to the cubic symmetry operator $S_{j}$ and such that $s_{j}^{0} \geqslant 0$. We can take

$$
\left\{\begin{array}{l}
s_{1} \stackrel{\text { def }}{=}(1,0,0,0), \quad s_{2} \stackrel{\text { def }}{=}(0,1,0,0), \\
s_{3} \stackrel{\text { def }}{=}(0,0,1,0), \quad s_{4} \stackrel{\text { def }}{=}(0,0,0,1), \\
s_{5} \stackrel{\text { def }}{=}(0.5,0.5,0.5,0.5), \\
s_{6} \stackrel{\text { def }}{=}(0.5,-0.5,0.5,0.5), \\
s_{7} \stackrel{\text { def }}{=}(0.5,0.5,-0.5,0.5), \\
s_{8} \stackrel{\text { def }}{=}(0.5,0.5,0.5,-0.5), \\
s_{9} \stackrel{\text { def }}{=}(0.5,-0.5,-0.5,0.5), \\
s_{10} \stackrel{\text { def }}{=}(0.5,-0.5,0.5,-0.5), \\
s_{11} \stackrel{\text { def }}{=}(0.5,0.5,-0.5,-0.5), \\
s_{12} \stackrel{\text { def }}{=}(0.5,-0.5,-0.5,-0.5), \\
s_{13} \stackrel{\text { def }}{=}(v, v, 0,0), \quad s_{14} \stackrel{\text { def }}{=}(v,-v, 0,0), \\
s_{15} \stackrel{\text { def }}{=}(v, 0, v, 0), \quad s_{16} \stackrel{\text { def }}{=}(v, 0,-v, 0), \\
s_{17} \stackrel{\text { def }}{=}(v, 0,0, v), \quad s_{18} \stackrel{\text { def }}{=}(v, 0,0,-v), \\
s_{19} \stackrel{\text { def }}{=}(0, v, v, 0), \quad s_{20} \stackrel{\text { def }}{=}(0, v, 0, v), \\
s_{21} \stackrel{\text { def }}{=}(0,0, v, v), \quad s_{22} \stackrel{\text { def }}{=}(0,-v, v, 0), \\
s_{23} \stackrel{\text { def }}{=}(0, v, 0,-v), \quad s_{24} \stackrel{\text { def }}{=}(0,0, v,-v), \\
v \stackrel{\text { def }}{=} \frac{1}{\sqrt{2}} .
\end{array}\right.
$$

The papers $[30,31]$ present methods for selecting single representations out of sets of symmetrically equivalent misorientations in the unit quaternion and Rodrigues vector spaces. The corresponding selection rules yield representations with minimum nonnegative rotation angles, i.e., with disorientation angles, and depend only on the considered symmetry of crystal lattices. Adopt the cubic lattice symmetry. Then, in the Rodrigues vector space, these rules lead to the compact domain

$$
\begin{aligned}
D_{\text {fund }} \stackrel{\text { def }}{=}\{ & \left(d_{1}, d_{2}, d_{3}\right) \in \mathbb{R}^{3}: \\
& \sqrt{2}-1 \geqslant d_{1} \geqslant d_{2} \geqslant d_{3} \geqslant 0, \\
& \left.d_{1}+d_{2}+d_{3} \leqslant 1\right\}
\end{aligned}
$$

which can be called the fundamental zone of misorientations subject to the cubic lattice symmetry. The subset of (32) determined by a fixed rotation angle $\theta \in[0, \pi)$ can be characterized 


$$
\begin{aligned}
D_{\text {fund }}^{\theta} \stackrel{\text { def }}{=}\left\{\left(d_{1}, d_{2}, d_{3}\right) \in \mathbb{R}^{3}:\right. \\
0 \leqslant d_{1} \leqslant \min \left(\tan \left(\frac{\theta}{2}\right), \sqrt{2}-1\right), \\
0 \leqslant d_{2} \leqslant \min \left(d_{1}, \sqrt{\tan ^{2}\left(\frac{\theta}{2}\right)-d_{1}^{2}}\right), \\
d_{3}=\sqrt{\tan ^{2}\left(\frac{\theta}{2}\right)-d_{1}^{2}-d_{2}^{2}}, \\
\left.d_{3} \leqslant \min \left(d_{2}, 1-d_{1}-d_{2}\right)\right\}
\end{aligned}
$$

We have

$$
\begin{array}{ll}
D_{\text {fund }}^{\theta} \neq \emptyset & \forall \theta \in\left[0, \theta_{\max }\right], \\
D_{\text {fund }}^{\theta}=\emptyset & \forall \theta>\theta_{\text {max }} .
\end{array}
$$

Also denote

$$
D_{\text {fund }}^{i} \stackrel{\text { def }}{=} \bigcup_{\theta \in\left[\theta_{i-1}, \theta_{i}\right)} D_{\text {fund }}^{\theta}, \quad i=\overline{1, r} .
$$

Note that, for every $i=\overline{1, r}$, an implementation $\left(d_{1}, d_{2}, d_{3}\right)$ of a random vector uniformly distributed on the set $D_{\text {fund }}^{i}$ can be generated as follows:

1) let $\theta$ be an implementation of a random variable with the uniform distribution on the interval $\left[\theta_{i-1}, \theta_{i}\right)$;

2) let $d_{1}$ be an implementation of a random variable with the uniform distribution on the interval $[0, \min (\tan (\theta / 2), \sqrt{2}-1)]$

3) let $d_{2}$ be an implementation of a random variable with the uniform distribution on the interval $\left[0, \min \left(d_{1}, \sqrt{\tan ^{2}(\theta / 2)-d_{1}^{2}}\right)\right]$;

4) take $d_{3}=\sqrt{\tan ^{2}(\theta / 2)-d_{1}^{2}-d_{2}^{2}}$ if $d_{3} \leqslant \min \left(d_{2}, 1-\right.$ $d_{1}-d_{2}$ ), otherwise make another attempt by starting from item 1 again.

The validity of this procedure can be directly verified by the use of (33),(35) and the following elementary result from probability theory (the latter should be applied several times).

Lemma. Let two random vectors $X_{1} \in \mathbb{R}^{n_{1}}, X_{2} \in \mathbb{R}^{n_{2}}$ constitute the random vector $\left(X_{1}, X_{2}\right) \in \mathbb{R}^{n_{1}+n_{2}}$ having the uniform distribution on some bounded set $G \subset \mathbb{R}^{n_{1}+n_{2}}$ with a positive Lebesgue measure meas $(G)$ in $\mathbb{R}^{n_{1}+n_{2}}$, i. e., the probability density of $\left(X_{1}, X_{2}\right)$ can be written as

$$
p_{\left(X_{1}, X_{2}\right)}(x)= \begin{cases}\frac{1}{\operatorname{meas}(G)}, & x \in G, \\ 0, & x \in \mathbb{R}^{n_{1}+n_{2}} \backslash G .\end{cases}
$$

Take a point $x_{1} \in \mathbb{R}^{n_{1}}$ at which the probability density of $X_{1}$ does not vanish:

$$
p_{X_{1}}\left(x_{1}\right)=\int_{\mathbb{R}^{n_{2}}} p_{\left(X_{1}, X_{2}\right)}\left(x_{1}, x_{2}\right) d x_{2}>0 .
$$

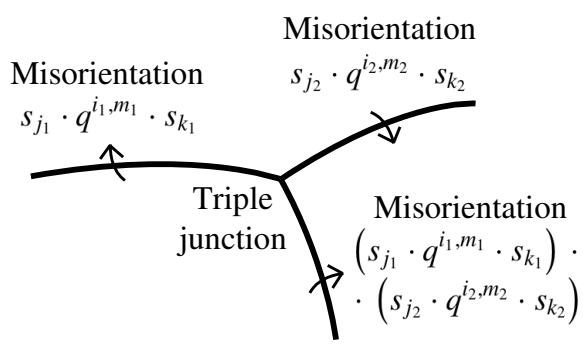

Figure 1: Misorientations of grain boundaries connected in a triple junction.

Then, at any point $x_{2} \in \mathbb{R}^{n_{2}}$, the conditional probability density of $X_{2}$ given the occurence of the value $x_{1}$ of $X_{1}$ is determined by

$$
\begin{aligned}
p_{X_{2} \mid X_{1}}\left(x_{2} \mid x_{1}\right) & =\frac{p_{\left(X_{1}, X_{2}\right)}\left(x_{1}, x_{2}\right)}{p_{X_{1}}\left(x_{1}\right)}= \\
& = \begin{cases}\frac{1}{\operatorname{meas}(G) \cdot p_{X_{1}}\left(x_{1}\right)}, & \left(x_{1}, x_{2}\right) \in G, \\
0, & \left(x_{1}, x_{2}\right) \in \mathbb{R}^{n_{1}+n_{2}} \backslash G,\end{cases}
\end{aligned}
$$

which corresponds to the uniform distribution on the set $\left\{x_{2} \in\right.$ $\left.\mathbb{R}^{n_{2}}:\left(x_{1}, x_{2}\right) \in G\right\}$.

Now we are ready to formulate the desired algorithm.

Step 1. Choose a sufficiently large number $M \in \mathbb{N}$, and, for every $i=\overline{1, r}$, generate $M$ implementations $d^{i, m} \stackrel{\text { def }}{=}$ $\left(d_{1}^{i, m}, d_{2}^{i, m}, d_{3}^{i, m}\right), m=\overline{1, M}$, of a random vector uniformly distributed on $D_{\text {fund }}^{i}$ (the corresponding procedure was described above).

Step 2. For every $i=\overline{1, r}, m=\overline{1, M}$, construct the quaternion

$$
q^{i, m} \stackrel{\text { def }}{=}\left(q_{0}^{i, m}, q_{0}^{i, m} \cdot d^{i, m}\right)
$$

with

$$
\begin{aligned}
& q_{0}^{i, m} \stackrel{\text { def }}{=} \cos \left(\frac{\theta^{i, m}}{2}\right), \\
& \theta^{i, m} \stackrel{\text { def }}{=} 2 \cdot \arctan \sqrt{\left(d_{1}^{i, m}\right)^{2}+\left(d_{2}^{i, m}\right)^{2}+\left(d_{3}^{i, m}\right)^{2}},
\end{aligned}
$$

based on the Rodrigues vector $d^{i, m}$. In fact, there is no need to apply the latter formula to compute the rotation angle $\theta^{i, m}$, because, according to the above-described procedure, $\theta^{i, m}$ is generated first and then used for generating $d_{1}^{i, m}, d_{2}^{i, m}, d_{3}^{i, m}$.

Step 3. Fix arbitrary indices $i_{1}$ and $i_{2} \geqslant i_{1}$ from the set $\{1,2, \ldots, r\}$. Let us show how to approximate the discrete distribution $\left\{\eta_{i_{1}, i_{2}}^{i}\right\}_{i=1}^{r}$. Our aim is to estimate possible disorientations of a grain boundary which is connected in a triple junction with two other boundaries having disorientations from $\left[\theta_{i_{1}-1}, \theta_{i_{1}}\right)$ and $\left[\theta_{i_{2}-1}, \theta_{i_{2}}\right)$. Take the symmetric equivalence into account and consider the following products of quaternions (see Figure 1):

$$
\begin{aligned}
& \left(s_{j_{1}} \cdot q^{i_{1}, m_{1}} \cdot s_{k_{1}}\right) \cdot\left(s_{j_{2}} \cdot q^{i_{2}, m_{2}} \cdot s_{k_{2}}\right)= \\
& =s_{j_{1}} \cdot q^{i_{1}, m_{1}} \cdot s_{l_{k_{1} j_{2}}} \cdot q^{i_{2}, m_{2}} \cdot s_{k_{2}}, \\
& m_{1}=\overline{1, M}, \quad m_{2}=\overline{1, M}, \\
& j_{1}=\overline{1,24}, \quad k_{1}=\overline{1,24}, \quad j_{2}=\overline{1,24}, \quad k_{2}=\overline{1,24} .
\end{aligned}
$$


By using matrix representations of rotations, cyclic permutation and similarity invariance of the trace of a matrix, as well as the expression for a rotation angle in terms of the trace of the corresponding rotation matrix, we conclude that, instead of (38), it suffices to consider

$$
\begin{gathered}
\left(s_{j_{1}}\right)^{-1} \cdot\left(s_{j_{1}} \cdot q^{i_{1}, m_{1}} \cdot s_{l_{k_{1} j_{2}}} \cdot q^{i_{2}, m_{2}} \cdot s_{k_{2}}\right) \cdot s_{j_{1}}= \\
=q^{i_{1}, m_{1}} \cdot s_{l_{k_{1} j_{2}}} \cdot q^{i_{2}, m_{2}} \cdot s_{l_{k_{2} j_{1}}}, \\
m_{1}=\overline{1, M}, \quad m_{2}=\overline{1, M}, \\
\left(i_{1}-1\right) \cdot M+m_{1} \leqslant\left(i_{2}-1\right) \cdot M+m_{2}, \\
j_{1}=\overline{1,24}, \quad k_{1}=\overline{1,24}, \quad j_{2}=\overline{1,24}, \quad k_{2}=\overline{1,24} .
\end{gathered}
$$

In turn, (39) can be characterized in a simpler way:

$$
\begin{aligned}
& q^{i_{1}, m_{1}} \cdot s_{j} \cdot q^{i_{2}, m_{2}} \cdot s_{k}, \\
& m_{1}=\overline{1, M}, \quad m_{2}=\overline{1, M}, \\
& \left(i_{1}-1\right) \cdot M+m_{1} \leqslant\left(i_{2}-1\right) \cdot M+m_{2}, \\
& j=\overline{1,24}, \quad k=\overline{1,24} .
\end{aligned}
$$

Then we come to the minimum nonnegative rotation angles

$$
\begin{aligned}
& \min _{k=1,24}\left(2 \cdot \arccos \left(q^{i_{1}, m_{1}} \cdot s_{j} \cdot q^{i_{2}, m_{2}} \cdot s_{k}\right)_{0}\right), \\
& m_{1}=\overline{1, M}, \quad m_{2}=\overline{1, M}, \\
& \left(i_{1}-1\right) \cdot M+m_{1} \leqslant\left(i_{2}-1\right) \cdot M+m_{2}, \\
& j=\overline{1,24}
\end{aligned}
$$

where $\left(q^{i_{1}, m_{1}} \cdot s_{j} \cdot q^{i_{2}, m_{2}} \cdot s_{k}\right)_{0}$ is the first component of the quaternion $q^{i_{1}, m_{1}} \cdot s_{j} \cdot q^{i_{2}, m_{2}} \cdot s_{k}$. There are

$$
\varkappa_{i_{1}, i_{2}} \stackrel{\text { def }}{=} \begin{cases}24 \cdot M^{2}, & i_{1} \neq i_{2}, \\ 24 \cdot \frac{M(M+1)}{2}=12 \cdot M(M+1), & i_{1}=i_{2},\end{cases}
$$

angles listed in (41); recall that $i_{1}, i_{2}$ are fixed there. For any $i=\overline{1, r}$, let $\varkappa_{i_{1}, i_{2}}^{i}$ denote the number of such angles from (41) that belong to the interval $\left[\theta_{i-1}, \theta_{i}\right)$. It remains to take

$$
\eta_{i_{2}, i_{1}}^{i}=\eta_{i_{1}, i_{2}}^{i} \approx \frac{\varkappa_{i_{1}, i_{2}}^{i}}{\varkappa_{i_{1}, i_{2}}}, \quad i=\overline{1, r} .
$$

\section{Representations of $P_{s,+}^{i}, P_{s,-}^{i}, P_{d, 1}^{i}, P_{d, 2}^{i}, i=\overline{1, r}$}

In order to construct representations of $P_{s,+}^{i}, P_{s,-}^{i}, P_{d, 1}^{i}, P_{d, 2}^{i}$, $i=\overline{1, r}$, we develop a modification of the approach proposed in [1, section 4]. First, let us briefly discuss the main features of the new framework.

1. The fiber-texture considerations in [1, section 4] allowed to write a direct representation for the misorientation of a grain boundary which is connected in a triple junction together with two other boundaries having given misorientations. In the general-texture case involving disorientation angles, such direct representations cannot be written, and the weights specified by the approximated discrete distributions $\left\{\eta_{i_{1}, i_{2}}^{i}\right\}_{i=1}^{r}, i_{1}=\overline{1, r}$, $i_{2}=\overline{1, r}$, have to be used.
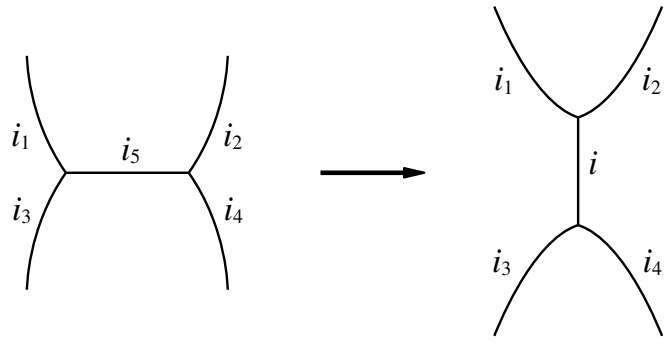

Figure 2: To formula (46) (creation of a grain boundary with a particular disorientation as a result of a neighbor switching).

2. The representations in [1, section 4] considered energy dissipation conditions in the sets of integration, i. e., topological transitions leading to an increase of the total grain boundary energy were supposed to be completely impossible. This caused such shortcoming of the developed kinetic model as discontinuities of the number- and length-weighted misorientation distribution functions with respect to the one-dimensional misorientation variable for some important types of grain boundary energy densities. As in the above-mentioned Monte Carlo techniques, let us consider the probabilities of energy-increasing topological transitions to be nonzero but proportional to an exponentially decaying factor similar to the Boltzmann weight. For this purpose, introduce the function

$$
\begin{aligned}
& \chi\left(\gamma_{\text {before }}, \gamma_{\text {after }}\right)= \\
& \quad= \begin{cases}1, & \gamma_{\text {after }} \leqslant \gamma_{\text {before }}, \\
\exp \left(-\frac{\gamma_{\text {after }}-\gamma_{\text {before }}}{\zeta \cdot \gamma_{\text {before }}}\right), & \gamma_{\text {after }} \geqslant \gamma_{\text {before }},\end{cases}
\end{aligned}
$$

where $\zeta$ is a positive parameter called effective "lattice temperature" (this is not a real physical temperature); see [13].

3. A significant remark should be added to the previous item. In common with [1, section 4], we consider a grain disappearance as a combination of two consecutive events. The first one is the neighbor switching which removes one zero-length boundary of a three-sided grain, generates a new zero-length boundary, and binds the remaining two boundaries of the destroyed grain into a two-sided grain. The subsequent event is the instantaneous removal of the generated zero-length boundary together with the whole constructed two-sided grain. Such interpretation is stipulated by the convention that, after a grain disappearance, a new triple junction is located at the first collision point; see, for instance, [28]. Let us treat the first of the mentioned events as described in item 3 , and let us not allow the second one to be energy-increasing. Note that the energy dissipation condition for the latter trivially holds when the grain boundary energy density fulfills the triangle inequality introduced in [23]. In particular, Read-Shockley and affine boundary energy densities satisfy this inequality; see [23].

Figures 2-5 illustrate the four types of events listed in the beginning of section 4. An index $j \in\{1,2, \ldots, r\}$ written near a grain boundary means that the corresponding disorientation angle belongs to the interval $\left[\theta_{j-1}, \theta_{j}\right)$. 

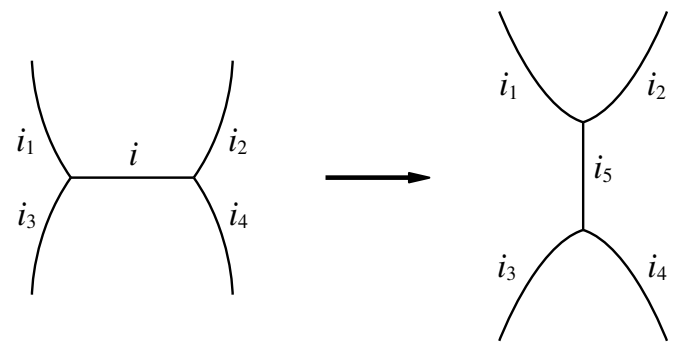

Figure 3: To formula (47) (removal of a grain boundary with a particular disorientation as a result of a neighbor switching).

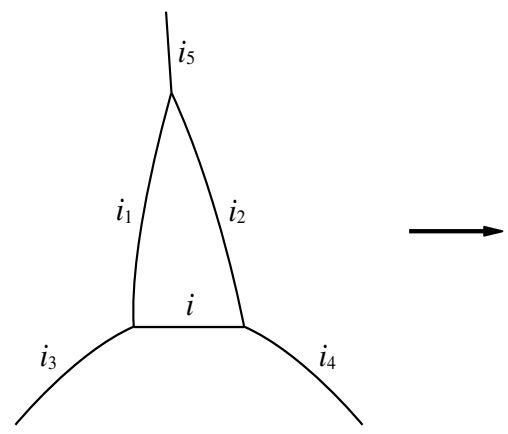

Figure 4: To formula (48) (removal of a zero-length grain boundary with a particular disorientation as a result of a grain disappearance).

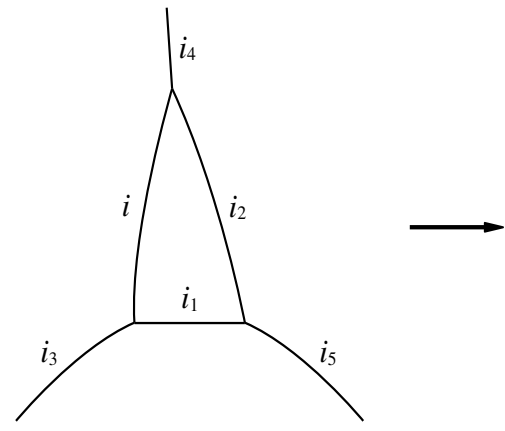

Figure 5: To formula (49) (removal of a nonzero-length grain boundary with a particular disorientation as a result of a grain disappearance).
According to Figures 2-5 (we focus on configurations before critical events) and the foregoing remarks, for all $i=\overline{1, r}$ and $N \in\left[N_{\min }, N_{0}\right]$, we can write

$$
\begin{aligned}
& P_{s,+}^{i}(N)=\frac{p_{s,+}^{i}(N)}{\sum_{j=1}^{r} p_{s,+}^{j}(N)}, \\
& P_{s,-}^{i}(N)=\frac{p_{s,-}^{i}(N)}{\sum_{j=1}^{r} p_{s,-}^{j}(N)}, \\
& P_{d, 1}^{i}(N)=\frac{p_{d, 1}^{i}(N)}{\sum_{j=1}^{r} p_{d, 1}^{j}(N)}, \\
& P_{d, 2}^{i}(N)=\frac{p_{d, 2}^{i}(N)}{\sum_{j=1}^{r} p_{d, 2}^{j}(N)},
\end{aligned}
$$

where

$$
\begin{aligned}
& p_{s,+}^{i}(N) \stackrel{\text { def }}{=} \eta_{i_{1}, i_{2}}^{i} \cdot \rho_{n}^{i_{1}}(N) \cdot \rho_{n}^{i_{2}}(N) \cdot \\
& \cdot \sum_{i_{5}=1}^{r} \chi\left(\gamma_{i_{5}}, \gamma_{i}\right) \cdot \rho_{n}^{i_{5}}(N) \text {. } \\
& \cdot\left(\sum_{i_{3}=1}^{r} \eta_{i_{5}, i_{1}}^{i_{3}} \cdot \rho_{n}^{i_{3}}(N)\right) \cdot\left(\sum_{i_{4}=1}^{r} \eta_{i_{5}, i_{2}}^{i_{4}} \cdot \rho_{n}^{i_{4}}(N)\right), \\
& p_{s,-}^{i}(N) \stackrel{\text { def }}{=} \rho_{n}^{i}(N) \cdot \rho_{n}^{i_{1}}(N) \cdot \rho_{n}^{i_{2}}(N) \cdot \\
& \cdot\left(\sum_{i_{3}=1}^{r} \eta_{i, i_{1}}^{i_{3}} \cdot \rho_{n}^{i_{3}}(N)\right) \cdot\left(\sum_{i_{4}=1}^{r} \eta_{i, i_{2}}^{i_{4}} \cdot \rho_{n}^{i_{4}}(N)\right) . \\
& \cdot\left(\sum_{i_{5}=1}^{r} \eta_{i_{1}, i_{2}}^{i_{5}} \cdot \chi\left(\gamma_{i}, \gamma_{i_{5}}\right)\right) \text {, } \\
& p_{d, 1}^{i}(N) \stackrel{\text { def }}{=} \rho_{n}^{i}(N) \cdot \rho_{n}^{i_{1}}(N) \cdot \rho_{n}^{i_{2}}(N) \cdot \\
& \cdot\left(\sum_{i_{3}=1}^{r} \eta_{i, i_{1}}^{i_{3}} \cdot \rho_{n}^{i_{3}}(N)\right) \cdot\left(\sum_{i_{4}=1}^{r} \eta_{i, i_{2}}^{i_{4}} \cdot \rho_{n}^{i_{4}}(N)\right) . \\
& \cdot\left(\sum_{\substack{i_{5} \in\{1,2, \ldots, r\}: \\
\gamma_{i_{5}} \leqslant \gamma_{i_{1}}+\gamma_{i_{2}}}} \eta_{i_{1}, i_{2}}^{i_{5}} \cdot \chi\left(\gamma_{i}, \gamma_{i_{5}}\right) \cdot \rho_{n}^{i_{5}}(N)\right) \text {, } \\
& p_{d, 2}^{i}(N) \stackrel{\text { def }}{=} \rho_{n}^{i}(N) \cdot \rho_{n}^{i_{1}}(N) \cdot \rho_{n}^{i_{2}}(N) \text {. } \\
& \cdot\left(\sum_{i_{3}=1}^{r} \eta_{i, i_{1}}^{i_{3}} \cdot \rho_{n}^{i_{3}}(N)\right) \cdot\left(\sum_{i_{5}=1}^{r} \eta_{i_{1}, i_{2}}^{i_{5}} \cdot \rho_{n}^{i_{5}}(N)\right) \text {. } \\
& \cdot\left(\sum_{\substack{i_{4} \in\{1,2, \ldots, r\}: \\
\gamma_{i_{4}} \leqslant \gamma_{i}+\gamma_{i_{2}}}} \eta_{i, i_{2}}^{i_{4}} \cdot \chi\left(\gamma_{i_{1}}, \gamma_{i_{4}}\right) \cdot \rho_{n}^{i_{4}}(N)\right) .
\end{aligned}
$$

As in $[1,22]$, we suppose the absence of correlations when writing the right-hand sides of (46)-(49). Besides, one can easily 
see that, if we denote

$$
\gamma_{\max } \stackrel{\text { def }}{=} \max _{\theta \in\left[0, \theta_{\max }\right]} \gamma(\theta)
$$

and replace $\gamma_{j}$ with the normalized value $\gamma_{j} / \gamma_{\max }$ for all $j=\overline{1, r}$, then the quantities (45) will remain the same.

\section{Remarks on the model's implementation}

1. Since we consider general textures with the cubic lattice symmetry, it is reasonable to take the initial number- and length-weighted DDFs following the well-known Mackenzie distribution. The latter appears when the orientation of every particular grain is chosen randomly in order to have the Euler angles (in Bunge convention)

$$
\left\{\begin{array}{l}
\varphi_{1}=2 \pi r_{1} \\
\Phi=\arccos \left(1-2 r_{2}\right), \\
\varphi_{2}=2 \pi r_{3}
\end{array}\right.
$$

where $r_{1}, r_{2}, r_{3}$ are independent random numbers generated according to the uniform distribution on the interval $[0,1]$; see, for instance, [13, 14, 23, 24].

2. Representations (46),(47) directly imply the relation

$$
\sum_{i=1}^{r} p_{s,+}^{i}(N)=\sum_{i=1}^{r} p_{s,-}^{i}(N) \quad \forall N \in\left[N_{\min }, N_{0}\right]
$$

complying with the property that every neighbor switching results in removal of a single boundary and creation of one new boundary; see also [1, section 5, remark 2].

3. As in [1, section 5, remark 3], we can prove that $\sum_{j=1}^{r} \rho_{n}^{j}(N)=1$ implies $\sum_{j=1}^{r} \rho_{n}^{j}(N-\Delta N)=1$ and that the solution to Cauchy problem (25),(27) should not take negative values if $\rho_{n, 0}^{j} \geqslant 0, j=\overline{1, r}$, and $\sum_{j=1}^{r} \rho_{n, 0}^{j}=1$.

4. Similarly to [1, section 5, remark 4], we can transform numerical scheme (28) in order to exclude negative values of an approximate solution to Cauchy problem (25),(27) which are possible in case of sufficiently large computational inaccura- cies. Finally, we come to the following scheme:

$$
\begin{aligned}
& \left\{\begin{array}{l}
\rho_{n}^{i}(N-\Delta N)= \\
\quad\left\{\begin{array}{l}
\rho_{n}(N) \cdot e^{A_{i}(N) \cdot \Delta N}+ \\
+\frac{B_{i}(N)}{A_{i}(N)} \cdot\left(e^{A_{i}(N) \cdot \Delta N}-1\right), \quad A_{i}(N) \neq 0,= \\
\rho_{n}^{i}(N)+B_{i}(N) \cdot \Delta N, \quad A_{i}(N)=0,
\end{array}\right.
\end{array}\right. \\
& =\rho_{n}^{i}(N) \cdot e^{A_{i}(N) \cdot \Delta N}+ \\
& +B_{i}(N) \cdot\left(\Delta N+\sum_{k=2}^{\infty} \frac{\left(A_{i}(N)\right)^{k-1} \cdot(\Delta N)^{k}}{k !}\right) \text {, } \\
& A_{i}(N) \stackrel{\text { def }}{=} \frac{1}{N} \cdot\left(1-\frac{\lambda(N)}{3} \cdot \frac{P_{s,-}^{i}(N)}{\rho_{n}^{i}(N)}-\right. \\
& \left.-\frac{1}{3} \cdot \frac{P_{d, 1}^{i}(N)}{\rho_{n}^{i}(N)}-\frac{2}{3} \cdot \frac{P_{d, 2}^{i}(N)}{\rho_{n}^{i}(N)}\right), \\
& B_{i}(N) \stackrel{\text { def }}{=} \frac{1}{N} \cdot \frac{\lambda(N)}{3} \cdot P_{s,+}^{i}(N), \\
& \Delta N>0 \text { is sufficiently small, } \\
& \rho_{n}^{i}\left(N_{0}\right)=\rho_{n, 0}^{i} .
\end{aligned}
$$

In comparison with (28), the a priori order of accuracy for (53) is at least not lower.

5. For estimating the ratio $\lambda$ of the neighbor switchings' rate to the grain disappearances' rate, let us use a heuristic method similar to the one proposed in [1, section 5, remark 5]. For every $N \in\left[N_{\min }, N_{0}\right]$, introduce the approximate average grain boundary energy density and fractions of low- and high-energy boundaries:

$$
\begin{aligned}
& \bar{\gamma}(N) \stackrel{\text { def }}{=} \sum_{i=1}^{r} \gamma_{i} \cdot \rho_{n}^{i}(N), \\
& \kappa_{\mathrm{le}}(N) \stackrel{\text { def }}{=} \sum_{\substack{i \in\{1,2, \ldots, r\}: \\
\gamma_{i} \leqslant \bar{\gamma}(N)}} \rho_{n}^{i}(N), \\
& \kappa_{\mathrm{he}}(N) \stackrel{\text { def }}{=} \sum_{\substack{i \in\{1,2, \ldots, r\}: \\
\gamma_{i} \geqslant \bar{\gamma}(N)}} \rho_{n}^{i}(N) \\
& \forall N \in\left[N_{\min }, N_{0}\right] .
\end{aligned}
$$

When $N=N_{0}$, i. e., $t=0$, we have

$$
\bar{\gamma}_{0} \stackrel{\text { def }}{=} \bar{\gamma}\left(N_{0}\right)=\sum_{i=1}^{r} \gamma_{i} \cdot \rho_{n, 0}^{i} .
$$

Assume that, in the isotropic case when $\gamma_{i}=\bar{\gamma}_{0}, i=\overline{1, r}$, the ratio $\lambda=\lambda(N)$ equals some constant $\lambda_{\text {isotr }}>0$ for all $N \in$ $\left[N_{\min }, N_{0}\right]$. As in [1], we will take $\lambda_{\text {isotr }} \approx 1.34$ according to the results of large-scale numerical simulations presented in [32]. For anisotropic grain boundary energy densities, we estimate $\lambda$ as

$$
\begin{array}{r}
\lambda(N) \approx \lambda_{\mathrm{isotr}} \cdot v\left(\frac{2 \cdot \kappa_{\mathrm{he}}(N)}{\kappa_{\mathrm{le}}(N)+\kappa_{\mathrm{he}}(N)}\right) \\
\forall N \in\left[N_{\mathrm{min}}, N_{0}\right],
\end{array}
$$

where $v:(0,2) \rightarrow(0,+\infty)$ is an increasing function such that $v(1)=1$; for instance, $v(x)=x$ for all $x \in(0,2)$. We can give 
the same reasons in favor of this estimate as in [1, section 5 , remark 5].

\section{Boundary lengthening models for estimating the length- weighted DDF}

In order to express $\rho_{l}^{i}, i=\overline{1, r}$, in terms of $\rho_{n}^{i}, i=\overline{1, r}$, via the second equality in (19), where we replace the dependence on $t$ with the dependence on $N$, let us apply three heuristic quantitative models which describe the mechanism of low-energy boundaries' lengthening at triple junctions. In section 9, we will compare these models with each other in terms of the obtained numerical results. Also note that the phenomenon of low-energy boundaries' lengthening was justified experimentally; see [16].

Let us denote the considered boundary lengthening models by $\mathbf{L}_{\mathbf{1}}, \mathbf{L}_{\mathbf{2}}, \mathbf{L}_{\mathbf{3}}$. They are stated as follows.

Model L $\mathbf{1}$ (see [10, 14]):

$$
\begin{aligned}
& \frac{1}{C_{1}(N)} \cdot \frac{\bar{L}\left(\theta_{i}^{\text {mid }}, N\right)}{\bar{L}(N)} \approx 1+c \cdot\left(1-\gamma_{i}\right) \\
& \forall N \in\left[N_{\text {min }}, N_{\text {interm }}\right], \quad i=\overline{1, r}
\end{aligned}
$$

under the condition that $\gamma_{\max }=1$, $c \approx 6$ is a suitable constant fitting parameter.

Model L $\mathbf{L}_{2}$ (see $\left.[1,15]\right)$ :

$$
\begin{aligned}
& \frac{1}{C_{2}(N)} \cdot \frac{\bar{L}\left(\theta_{i}^{\text {mid }}, N\right)}{\bar{L}(N)} \approx \\
& \approx \begin{cases}1+\frac{1}{2}\left(1-\frac{\sqrt{3}}{\tan \left(\arccos \left(\frac{\gamma_{i}}{2 \bar{\gamma}(N)}\right)\right)}\right), & \frac{\gamma_{i}}{\bar{\gamma}(N)} \leqslant \sqrt{3}, \\
0, & \frac{\gamma_{i}}{\bar{\gamma}(N)} \geqslant \sqrt{3},\end{cases} \\
& \forall N \in\left[N_{\text {min }}, N_{\text {interm }}\right], \quad i=\overline{1, r} .
\end{aligned}
$$

Model L $\mathbf{L}_{3}$ (see [14]):

$$
\begin{aligned}
& \frac{1}{C_{3}(N)} \cdot \frac{\bar{L}\left(\theta_{i}^{\mathrm{mid}}, N\right)}{\bar{L}(N)} \approx \\
& \approx a_{0}+a_{1} \cdot \frac{\gamma_{i}}{\gamma_{\max }}+a_{2} \cdot\left(\frac{\gamma_{i}}{\gamma_{\max }}\right)^{2} \\
& \forall N \in\left[N_{\min }, N_{\text {interm }}\right], \quad i=\overline{1, r}, \\
& a_{0}=2.345, \quad a_{1}=-1.592, \quad a_{2}=0.2231 .
\end{aligned}
$$

Here $N_{\text {interm }} \in\left(N_{\min }, N_{0}\right)$ is some constant; for instance, $N_{\text {interm }}=\left(N_{\min }+N_{0}\right) / 2$. Its presence is stipulated by the fact that, for anisotropic grain boundary energy densities and the Mackenzie initial number- and length-weighted DDFs (see remark 1 in section 7), relations (57)-(59) are not expected to hold at initial stages of texture evolutions. Furthermore, $C_{1}(N), C_{2}(N), C_{3}(N)$ are normalization quantities for all $N \in$ [ $\left.N_{\text {min }}, N_{\text {interm }}\right]$. Nevertheless, after rewriting the right-hand side of the second equality in (19) via any of relations (57)-(59), the normalization quantities cancel each other out in the numerator and denominator. Indeed, (19) automatically implies (20).

According to [10, 12, 23, 25, 28], higher-order junctions such as quadruple junctions may become stable in case of highly anisotropic boundary energy densities. The current generaltexture framework as well as the fiber-texture framework from [1] do not explicitly operate with stable junctions of orders greater than 3 . However, as in [23, 28], it is possible to interpret a quadruple junction as two triple junctions connected through a common grain boundary with a relatively small length.

\section{Numerical simulations}

We adopt the cubic lattice symmetry for which (8) holds. The grid (14) is chosen as follows:

$$
\begin{aligned}
& \theta_{i}=i \cdot 2 \cdot \frac{\pi}{180}=i \cdot 2^{\circ}, \quad i=\overline{0, r}, \quad r=32, \\
& \tilde{\theta}_{\max }=64 \cdot \frac{\pi}{180}=64^{\circ} .
\end{aligned}
$$

The a priori distributions $\left\{\eta_{i_{1}, i_{2}}^{i}\right\}_{i=1}^{r}, i_{1}=\overline{1, r}, i_{2}=\overline{1, r}$, are estimated by the use of the algorithm described in section 5 . They have to be constructed only once for the cubic or any other particular lattice symmetry (unlike the number- and lengthweighted DDFs depending also on other parameters and initial conditions). The corresponding nonnormalized histograms for several pairs $\left(i_{1}, i_{2}\right)$ are shown in Figure 6.

Six types of grain boundary energy densities are considered; see Figures 7-12.

Type I. Isotropic boundary energy density:

$$
\gamma_{\mathrm{I}}(\theta) \stackrel{\text { def }}{\equiv} \gamma_{m}>0 \quad \forall \theta \in\left[0, \theta_{\max }\right] .
$$

Type II. Read-Shockley boundary energy density (see [1, 10, $13,18,23,24])$ with the cutoff angle $\theta_{\mathrm{II}}=15 \cdot \pi / 180=15^{\circ}$ :

$$
\begin{aligned}
& \gamma_{\mathrm{II}}(\theta) \stackrel{\text { def }}{=} \\
& \stackrel{\text { def }}{=} \begin{cases}\gamma_{l}, & \theta=0, \\
\gamma_{l}+\left(\gamma_{m}-\gamma_{l}\right) \cdot \frac{\theta}{\theta_{\mathrm{II}}} \cdot\left(1-\ln \left(\frac{\theta}{\theta_{\mathrm{II}}}\right)\right), & \theta \in\left(0, \theta_{\mathrm{II}}\right], \\
\gamma_{m}, & \theta \geqslant \theta_{\mathrm{II}},\end{cases} \\
& \forall \theta \in\left[0, \theta_{\max }\right], \\
& \gamma_{l} \in\left[0, \gamma_{m}\right) .
\end{aligned}
$$

Type III. Read-Shockley boundary energy density with the cutoff angle $\theta_{\mathrm{III}}=45 \cdot \pi / 180=45^{\circ}: \gamma_{\mathrm{III}}=\gamma_{\mathrm{III}}(\theta)$ is defined by (62) if we replace $\theta_{\text {II }}$ with $\theta_{\text {III }}$ there.

Type IV. Read-Shockley boundary energy density with the cutoff angle $\theta_{\mathrm{IV}}=\theta_{\max } \approx 62.8 \cdot \pi / 180=62.8^{\circ}: \gamma_{\mathrm{IV}}=\gamma_{\mathrm{IV}}(\theta)$ is defined by (62) if we replace $\theta_{\mathrm{II}}$ with $\theta_{\mathrm{IV}}$ there.

Type V. Affine boundary energy density (see [23]):

$$
\gamma_{\mathrm{V}}(\theta) \stackrel{\text { def }}{=} \gamma_{l}+\left(\gamma_{m}-\gamma_{l}\right) \cdot \frac{\theta}{\theta_{\max }} \quad \forall \theta \in\left[0, \theta_{\max }\right] .
$$


$\mathrm{i}_{1}=\mathrm{i}_{2}=1$

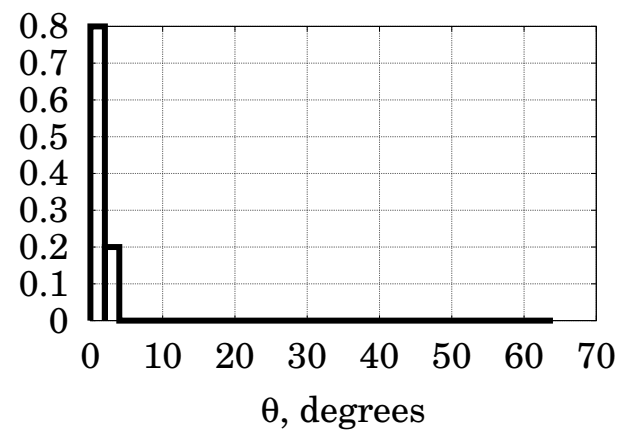

$\mathrm{i}_{1}=5, \mathrm{i}_{2}=32$
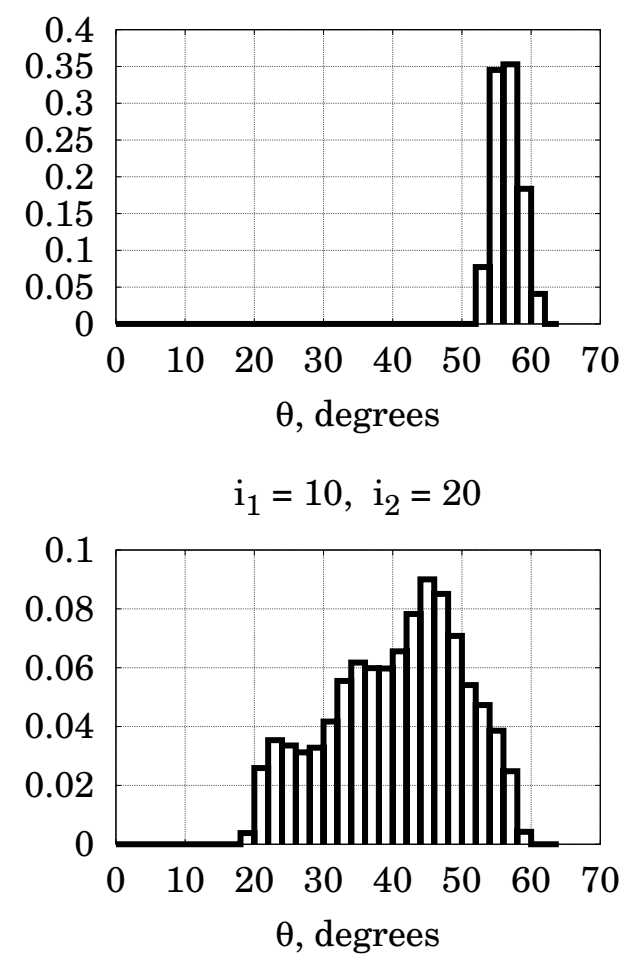

$\mathrm{i}_{1}=\mathrm{i}_{2}=16$

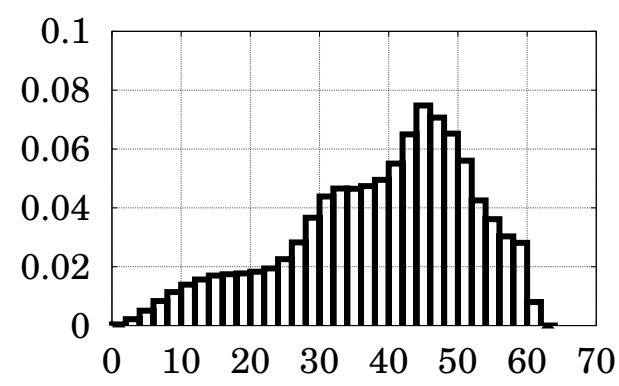

$\theta$, degrees

Figure 6: Nonnormalized histograms for the a priori disorientation distributions corresponding to $\left\{\eta_{i_{1}, i_{2}}^{i}\right\}_{i=1}^{r}$ for different pairs $\left(i_{1}, i_{2}\right)$.

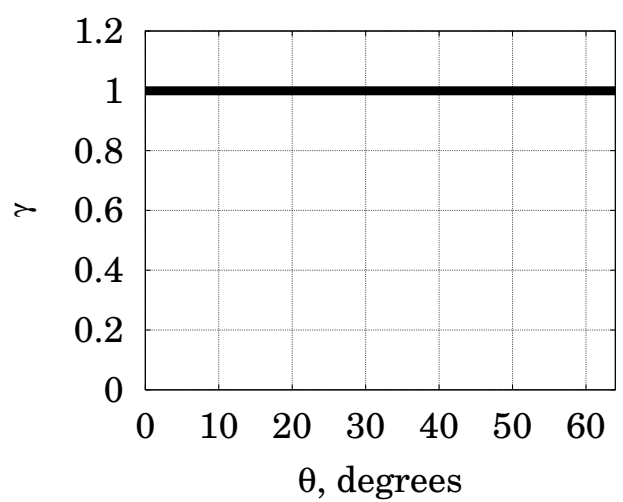

Figure 7: Grain boundary energy density of Type $\mathbf{I}$.

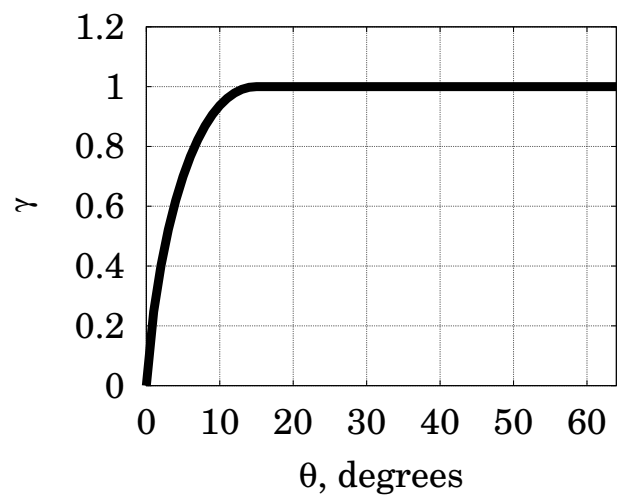

Figure 8: Grain boundary energy density of Type II.

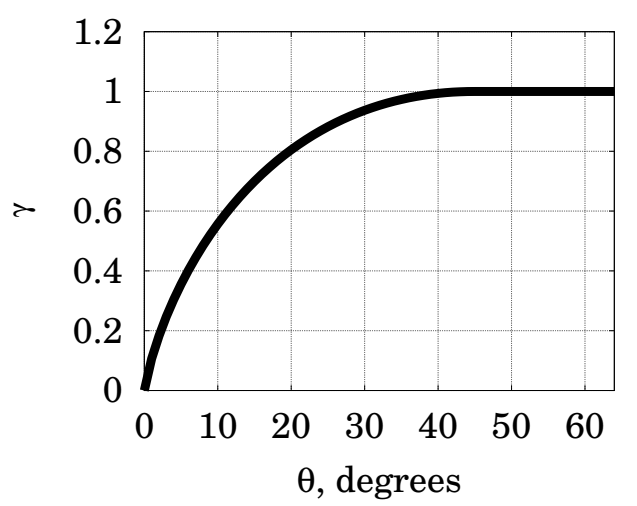

Figure 9: Grain boundary energy density of Type III. 


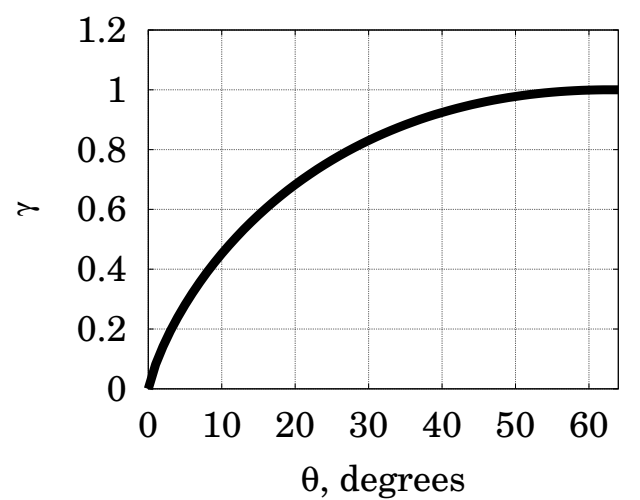

Figure 10: Grain boundary energy density of Type IV.

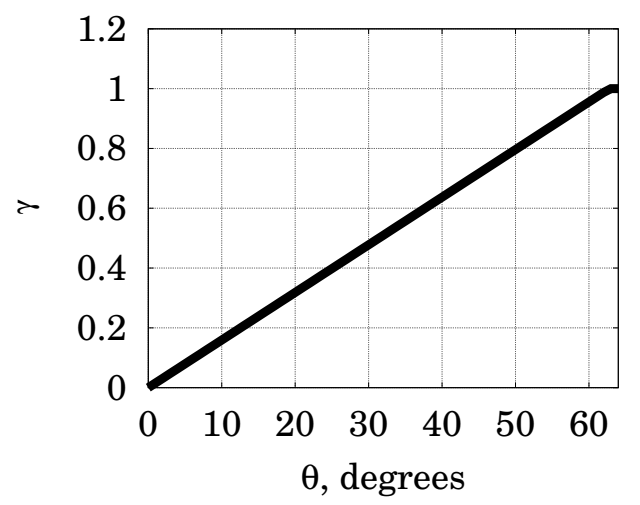

Figure 11: Grain boundary energy density of Type V.

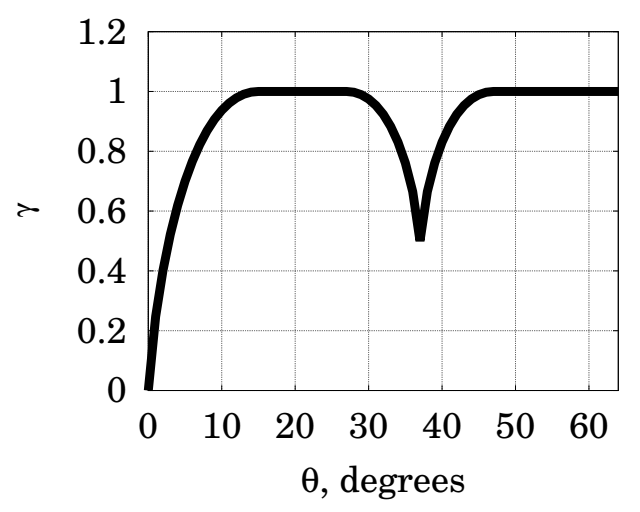

Figure 12: Grain boundary energy density of Type VI.
Type VI. Modification of $\gamma_{\mathrm{II}}$ with an additional local minimum (see $[1,18])$ :

$$
\begin{aligned}
& \gamma_{\mathrm{VI}}(\theta) \stackrel{\text { def }}{=} \\
& \stackrel{\text { def }}{=}\left\{\begin{array}{r}
\gamma_{\mathrm{II}}(\theta), \quad \theta \in\left[0, \theta_{\mathrm{VI}}-\theta_{\mathrm{VI}}^{\prime}\right], \\
\gamma_{l, 1}+\left(\gamma_{m}-\gamma_{l, 1}\right) \cdot \frac{\left|\theta-\theta_{\mathrm{VI}}\right|}{\theta_{\mathrm{VI}}^{\prime}} . \\
\cdot\left(1-\ln \left(\frac{\left|\theta-\theta_{\mathrm{VI}}\right|}{\theta_{\mathrm{VI}}^{\prime}}\right)\right),
\end{array}\right. \\
& \theta \in\left[\theta_{\mathrm{VI}}-\theta_{\mathrm{VI}}^{\prime}, \theta_{\mathrm{VI}}+\theta_{\mathrm{VI}}^{\prime}\right] \backslash\left\{\theta_{\mathrm{VI}}\right\} \text {, } \\
& \gamma_{l, 1}, \quad \theta=\theta_{\mathrm{VI}}, \\
& \gamma_{m}, \quad \theta \geqslant \theta_{\mathrm{VI}}+\theta_{\mathrm{VI}}^{\prime},
\end{aligned}
$$

$\forall \theta \in\left[0, \theta_{\max }\right]$,

$\gamma_{l, 1} \in\left(\gamma_{l}, \gamma_{m}\right), \quad \theta_{\mathrm{VI}} \in\left(\theta_{\mathrm{II}}, \theta_{\mathrm{max}}\right], \quad \theta_{\mathrm{VI}}^{\prime} \in\left(0, \theta_{\mathrm{VI}}-\theta_{\mathrm{II}}\right]$.

Here we take $\theta_{\mathrm{VI}}=37 \cdot \pi / 180=37^{\circ}, \theta_{\mathrm{VI}}^{\prime}=10 \cdot \pi / 180=10^{\circ}$, $\gamma_{l, 1}=\left(\gamma_{l}+\gamma_{m}\right) / 2$.

It is obvious that, for any fixed constant $C>0$, replacing $\gamma(\theta)$ with $C \cdot \gamma(\theta)$ does not change our approximations of $\left\{\rho_{n}^{i}(N)\right\}_{i=1}^{r}$, $\left\{\rho_{l}^{i}(N)\right\}_{i=1}^{r}$. Let us choose $\gamma_{l}=0, \gamma_{m}=1$.

In conformity with [13, subsection 2.2], the effective "lattice temperature" is taken as $\zeta=0.7$ for two-dimensional simulations.

We set $N_{0}=300000$. This is reasonable if the initial number of grains is 100000 and the initial average number of sides per grain is 6 , because each grain boundary belongs to exactly two grains; see, for instance, [9, section 4.6.2]. According to remark 1 in section 7, $\left\{\rho_{n, 0}^{i}\right\}_{i=1}^{r}=\left\{\rho_{n}^{i}\left(N_{0}\right)\right\}_{i=1}^{r}$ and $\left\{\rho_{l, 0}^{i}\right\}_{i=1}^{r}=\left\{\rho_{l}^{i}\left(N_{0}\right)\right\}_{i=1}^{r}$ follow the nonnormalized histograms for Mackenzie distribution on the disorientation grid (60). In order to compute $\left\{\rho_{n}^{i}(N)\right\}_{i=1}^{r}$, we use numerical scheme (53) with $\Delta N=N_{0} / 600=500$ step-by-step till the very end $N_{\min }=0$ at which $\left.\left\{\rho_{n}^{i}\right\}_{i=1}^{r}\right|_{N=0}$ is obtained by means of $\left.\left\{\rho_{n}^{i}\right\}_{i=1}^{r}\right|_{N=\Delta N}$. After approximating $\left\{\rho_{n}^{i}(N)\right\}_{i=1}^{r}$, we apply (57)-(59) with $N_{\text {interm }}=$ $N_{0} / 2=150000$ so as to estimate $\left\{\rho_{l}^{i}(N)\right\}_{i=1}^{r}$ from $N=N_{\text {interm }}$ to $N=0$.

As in [1], we consider the following two ways of determining the ratio $\lambda=\lambda(N)$ of the neighbor switchings' rate to the grain disappearances' rate (see remark 5 in section 7 and recall that the value $\lambda_{\text {isotr }}=1.34$ is taken from [32]):

А) $\lambda(N) \equiv 1.34$

B) $\lambda=\lambda(N)$ is estimated according to (56) with $\lambda_{\text {isotr }}=1.34$ and $v(x) \equiv x$.

Nevertheless, we will focus on case $\mathbf{B}$, because it is nontrivial and, as will be seen in several examples below, there is only a slight difference between cases $\mathbf{A}, \mathbf{B}$ in terms of the constructed approximations of the number- and length-weighted DDFs; also recall [1, section 5, the end of remark 5].

Figures 13-22 show the evolving normalized histograms based on $\left\{\rho_{n}^{i}(N)\right\}_{i=1}^{r}$ and on the three variants of $\left\{\rho_{l}^{i}(N)\right\}_{i=1}^{r}$ estimated via boundary lengthening models $\mathbf{L}_{\mathbf{1}}, \mathbf{L}_{\mathbf{2}}, \mathbf{L}_{\mathbf{3}}$. In Figures 13-18, case $\mathbf{B}$ is illustrated for all the considered types 


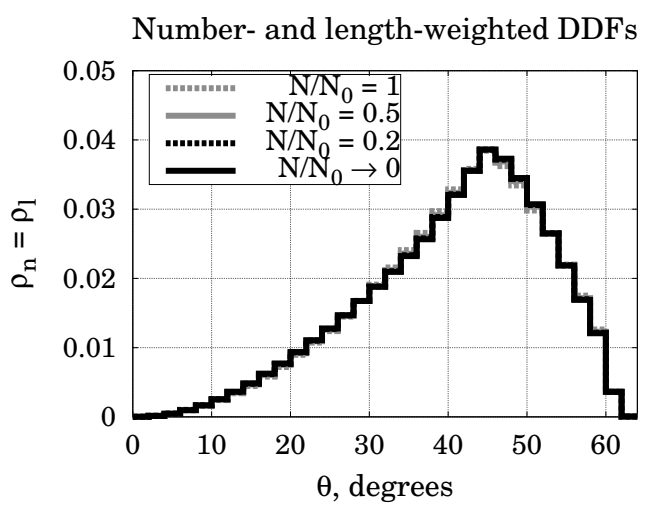

Figure 13: Normalized histograms approximating the number- and lengthweighted DDFs for the grain boundary energy density of Type $\mathbf{I}$ and $\lambda=\lambda(N)$ determined by (56) with $\lambda_{\text {isotr }}=1.34, v(x) \equiv x$.

of boundary energy densities. The numerical results in case $\mathbf{A}$ turn out to be negligibly different from those in case $\mathbf{B}$. For the boundary energy densities of Types III-VI, one can directly see this by comparing Figures 15-18 with Figures 19-22, respectively. For the boundary energy densities of Types I,II, there is almost no evolution (as follows from Figures 13,14), and the similarity between the portraits in cases $\mathbf{A}, \mathbf{B}$ is even more evident; the corresponding figures for case $\mathbf{A}$ are omitted for the sake of brevity.

Figures 23-25 illustrate the graphs of the right-hand sides of representations (57)-(59) (specifying boundary lengthening models $\mathbf{L}_{\mathbf{1}}, \mathbf{L}_{\mathbf{2}}, \mathbf{L}_{\mathbf{3}}$ ) for the boundary energy densities of Types IV-VI. The right-hand side of (58) depending on the average boundary energy density $\bar{\gamma}(N)$ as well as on the disorientation variable $\theta$ is computed for case $\mathbf{B}$.

Figures 26-29 show the evolving normalized histograms based on $\left\{P_{s,+}^{i}(N)\right\}_{i=1}^{r},\left\{P_{s,-}^{i}(N)\right\}_{i=1}^{r},\left\{P_{d, 1}^{i}(N)\right\}_{i=1}^{r},\left\{P_{d, 2}^{i}(N)\right\}_{i=1}^{r}$ and graphs of the functions (54),(56) for the boundary energy densities of Types II,VI and case $\mathbf{B}$.

In addition to the mentioned similarity of cases $\mathbf{A}, \mathbf{B}$, the following principal conclusions can be drawn from the presented simulation results.

1. With the help of the factor (44), we have avoided unnatural jumps of the approximated number- and length-weighted DDFs at cutoff disorientation angles; see Figures 14-16,1820,22 . Such jumps do not appear in the large-scale simulation results demonstrated in $[13,18,23]$ and, therefore, constitute a shortcoming of the model developed in [1].

2. Figures 13-16,19,20 indicate that, for the boundary energy densities of Types $\mathbf{I}-\mathbf{I V}$, the number-weighted DDF evolves negligibly and do not differ substantially from Mackenzie distribution. This conforms with the Monte Carlo simulation results shown in [13, Figure 5(a)]. From Figures 17,18,21,22 concerning the boundary energy densities of Types $\mathbf{V}, \mathbf{V I}$, we observe a noticeable increase in the number-weighted DDF on some of low-energy disorientation intervals. However, this increase is much weaker than in the fiber-texture case, where large peaks of misorientation distributions appear at points of

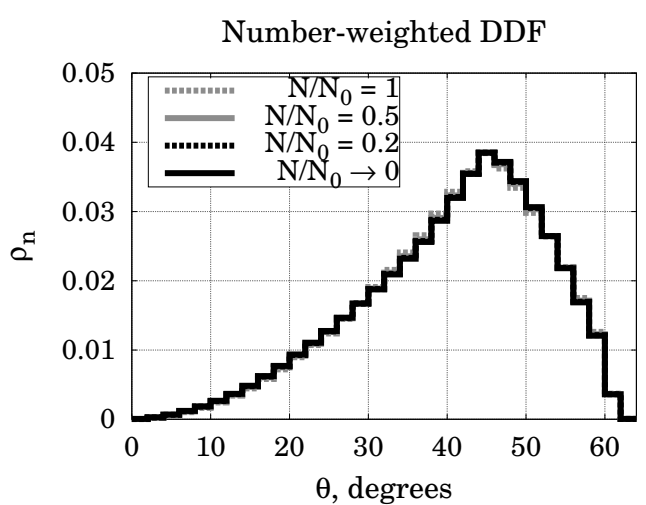

Length-weighted DDF, model $\mathrm{L}_{1}$

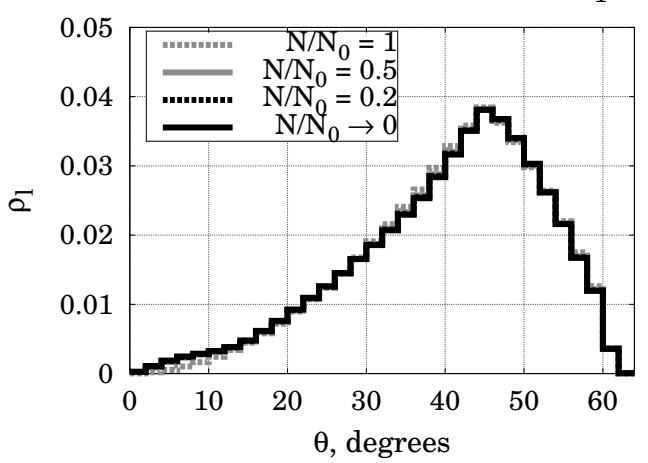

Length-weighted DDF, model $\mathrm{L}_{2}$

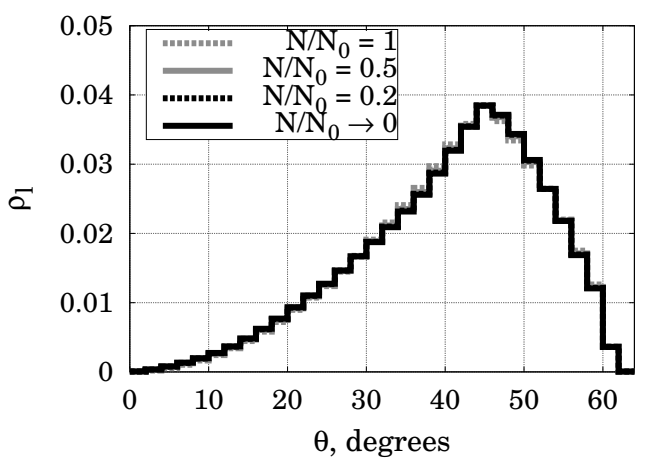

Length-weighted DDF, model $\mathrm{L}_{3}$

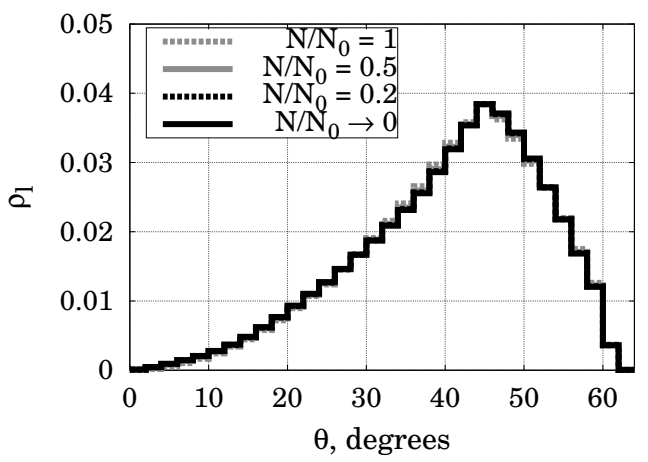

Figure 14: Normalized histograms approximating the number- and lengthweighted DDFs for the grain boundary energy density of Type II and $\lambda=\lambda(N)$ determined by (56) with $\lambda_{\text {isotr }}=1.34, v(x) \equiv x$. Three different boundary lengthening models $\mathbf{L}_{\mathbf{1}}, \mathbf{L}_{\mathbf{2}}, \mathbf{L}_{\mathbf{3}}$ for representing the length-weighted DDF in terms of the number-weighted DDF are implemented. 


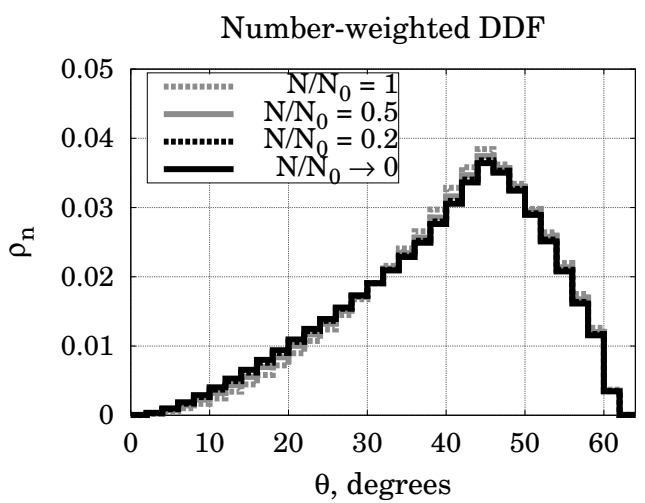

Length-weighted DDF, model $\mathrm{L}_{1}$

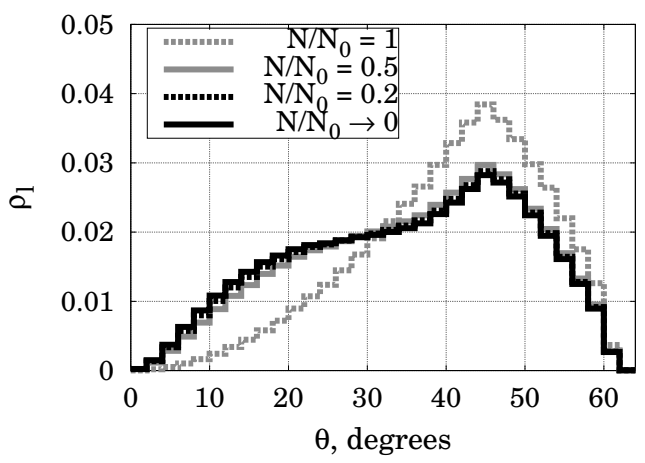

Length-weighted DDF, model $\mathrm{L}_{2}$

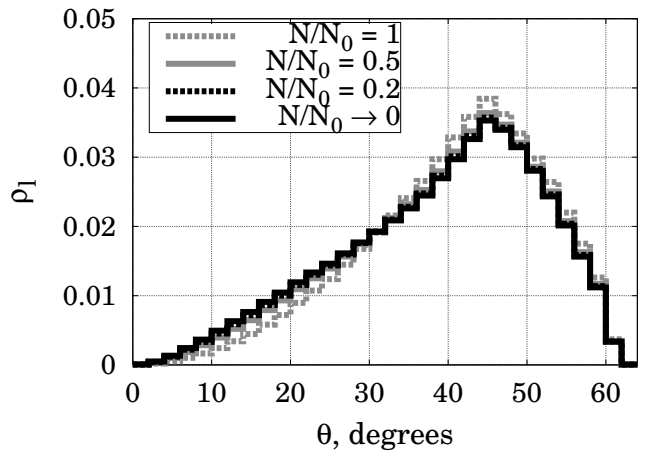

Length-weighted DDF, model $\mathrm{L}_{3}$

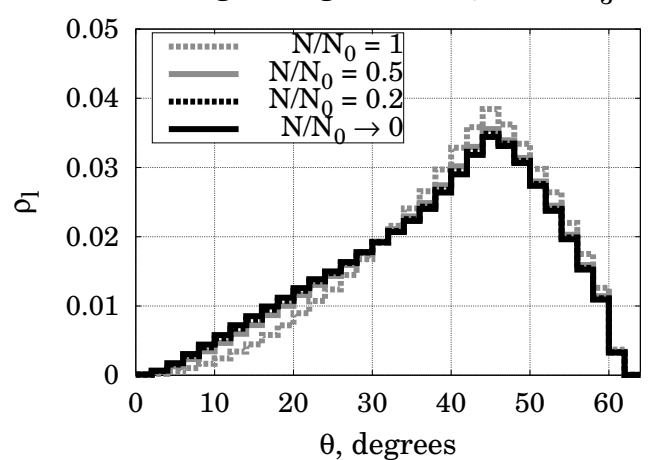

Figure 15: Normalized histograms approximating the number-and lengthweighted DDFs for the grain boundary energy density of Type III and $\lambda=\lambda(N)$ determined by (56) with $\lambda_{\text {isotr }}=1.34, v(x) \equiv x$. Three different boundary lengthening models $\mathbf{L}_{\mathbf{1}}, \mathbf{L}_{\mathbf{2}}, \mathbf{L}_{\mathbf{3}}$ for representing the length-weighted $D D F$ in terms of the number-weighted DDF are implemented.

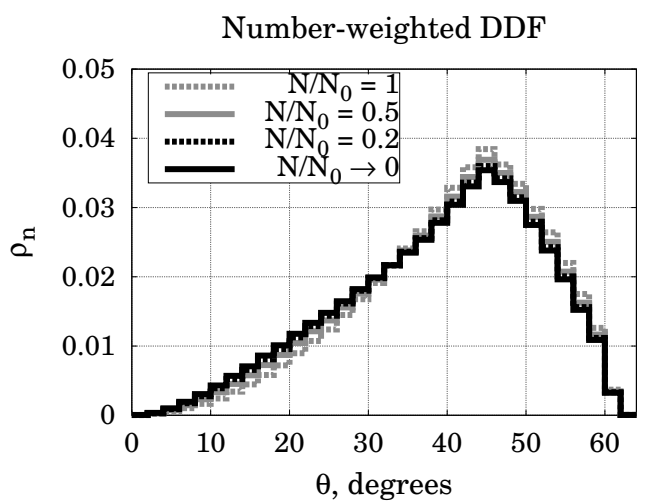

Length-weighted DDF, model $\mathrm{L}_{1}$

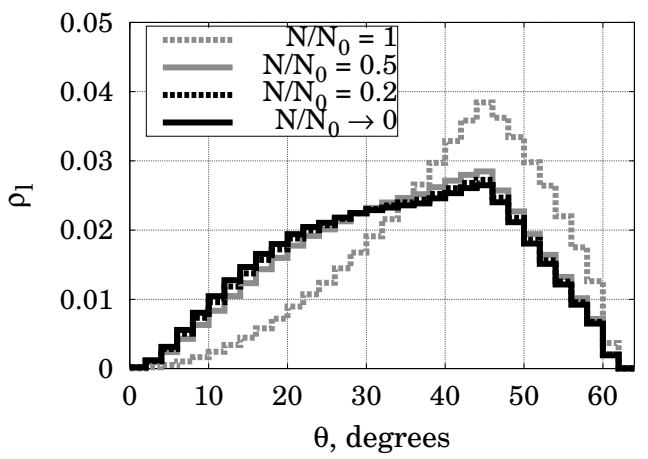

Length-weighted DDF, model $\mathrm{L}_{2}$

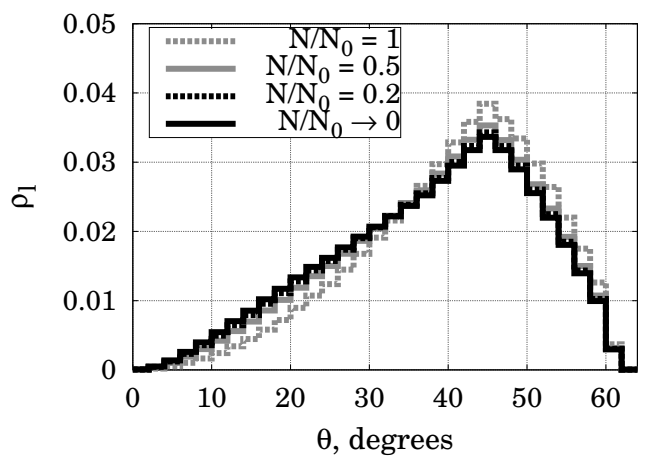

Length-weighted DDF, model $\mathrm{L}_{3}$

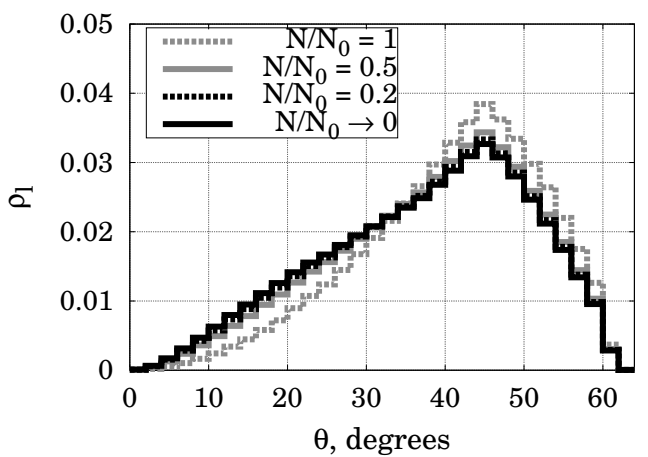

Figure 16: Normalized histograms approximating the number- and lengthweighted DDFs for the grain boundary energy density of Type $\mathbf{I V}$ and $\lambda=\lambda(N)$ determined by (56) with $\lambda_{\text {isotr }}=1.34, v(x) \equiv x$. Three different boundary lengthening models $\mathbf{L}_{\mathbf{1}}, \mathbf{L}_{\mathbf{2}}, \mathbf{L}_{\mathbf{3}}$ for representing the length-weighted $D D F$ in terms of the number-weighted DDF are implemented. 


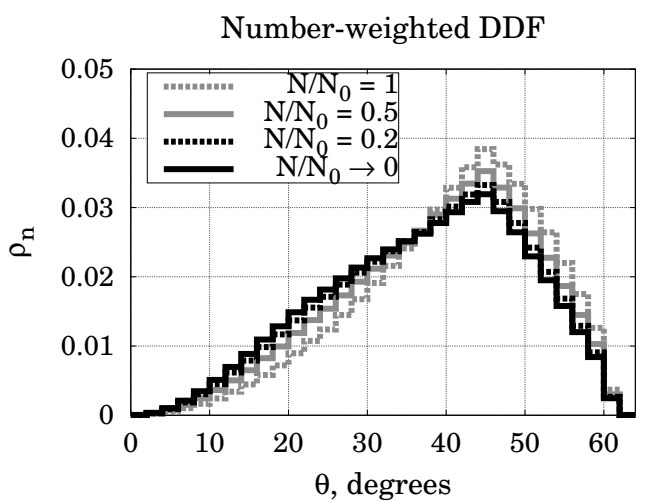

Length-weighted DDF, model $\mathrm{L}_{1}$

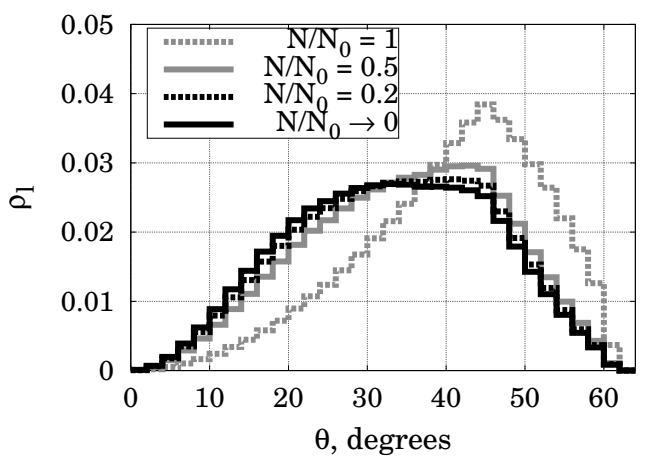

Length-weighted DDF, model $\mathrm{L}_{2}$

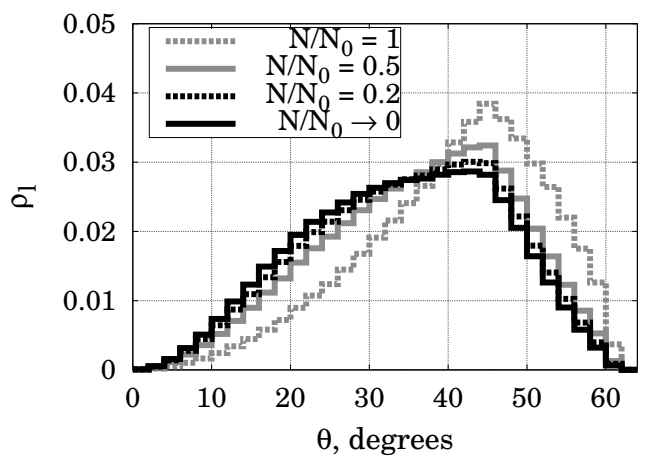

Length-weighted DDF, model $\mathrm{L}_{3}$

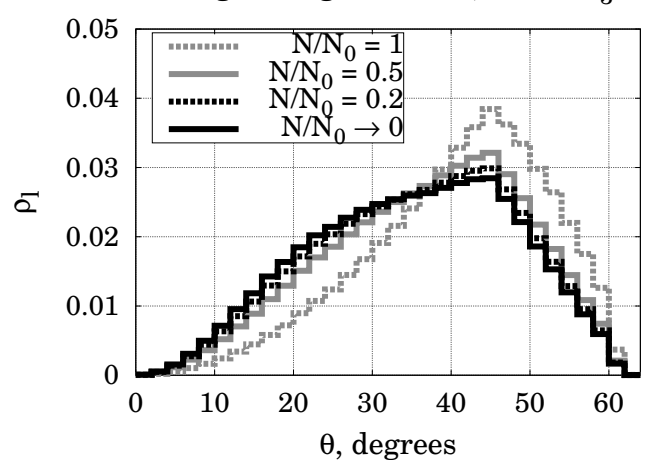

Figure 17: Normalized histograms approximating the number-and lengthweighted DDFs for the grain boundary energy density of Type $\mathbf{V}$ and $\lambda=\lambda(N)$ determined by (56) with $\lambda_{\text {isotr }}=1.34, v(x) \equiv x$. Three different boundary lengthening models $\mathbf{L}_{\mathbf{1}}, \mathbf{L}_{\mathbf{2}}, \mathbf{L}_{\mathbf{3}}$ for representing the length-weighted $D D F$ in terms of the number-weighted DDF are implemented.

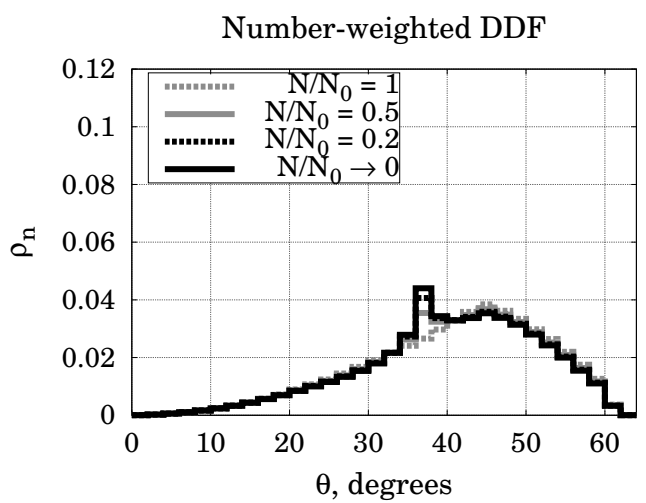

Length-weighted DDF, model $\mathrm{L}_{1}$

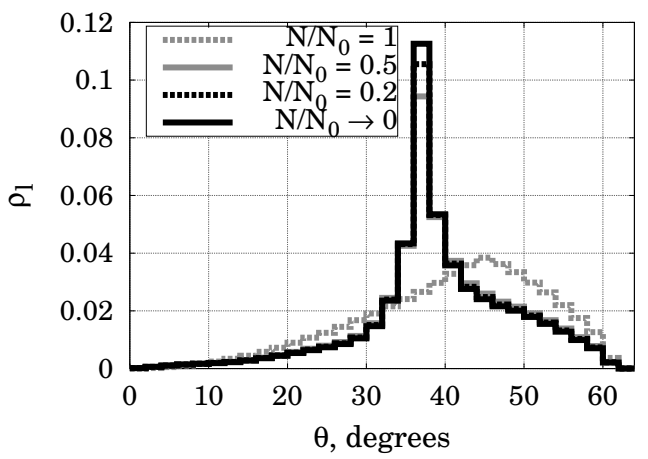

Length-weighted DDF, model $\mathrm{L}_{2}$

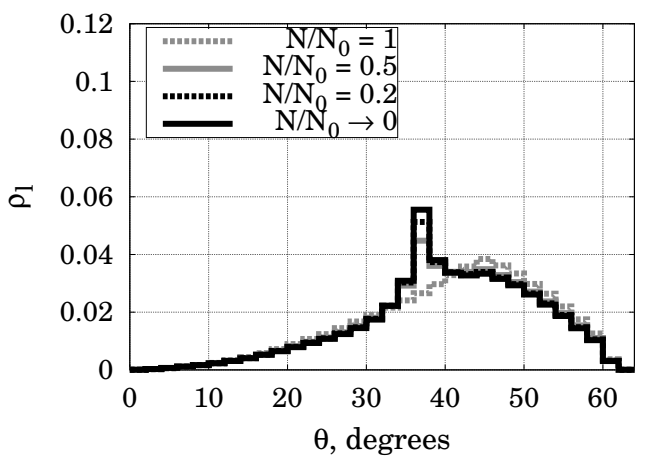

Length-weighted DDF, model $\mathrm{L}_{3}$

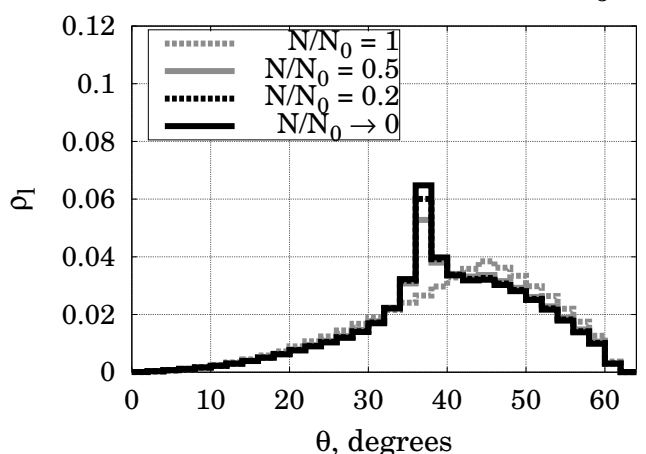

Figure 18: Normalized histograms approximating the number- and lengthweighted DDFs for the grain boundary energy density of Type $\mathbf{V I}$ and $\lambda=\lambda(N)$ determined by (56) with $\lambda_{\text {isotr }}=1.34, v(x) \equiv x$. Three different boundary lengthening models $\mathbf{L}_{\mathbf{1}}, \mathbf{L}_{\mathbf{2}}, \mathbf{L}_{\mathbf{3}}$ for representing the length-weighted $D D F$ in terms of the number-weighted DDF are implemented. 


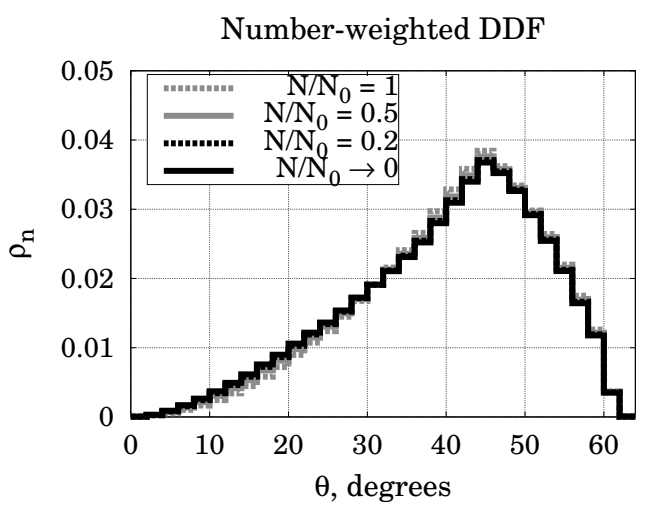

Length-weighted DDF, model $\mathrm{L}_{1}$

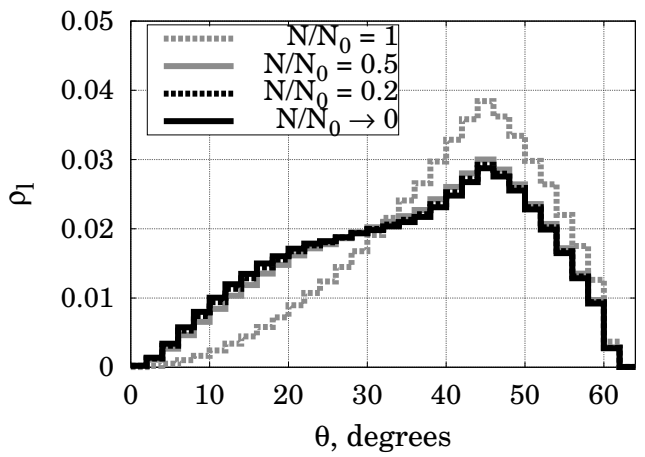

Length-weighted DDF, model $\mathrm{L}_{2}$

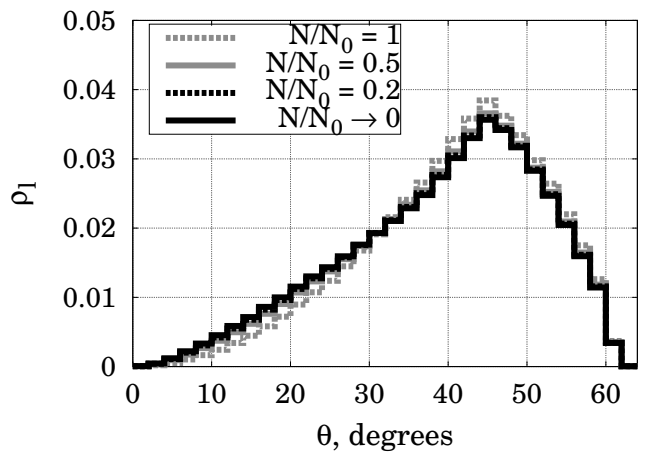

Length-weighted DDF, model $\mathrm{L}_{3}$

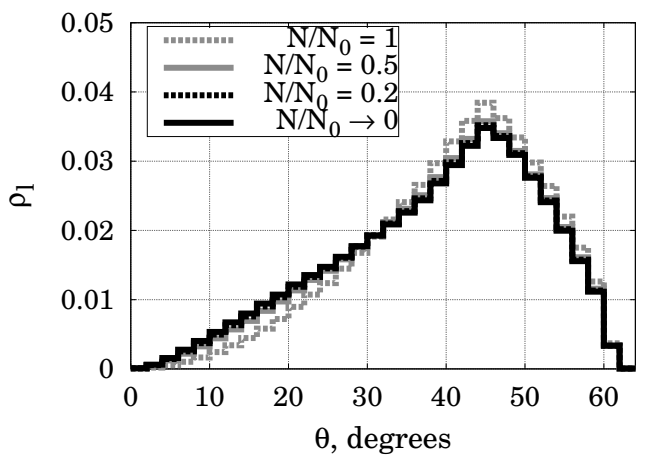

Figure 19: Normalized histograms approximating the number-and lengthweighted DDFs for the grain boundary energy density of Type III and $\lambda(N) \equiv$ 1.34. Three different boundary lengthening models $\mathbf{L}_{\mathbf{1}}, \mathbf{L}_{\mathbf{2}}, \mathbf{L}_{\mathbf{3}}$ for representing the length-weighted DDF in terms of the number-weighted DDF are implemented.

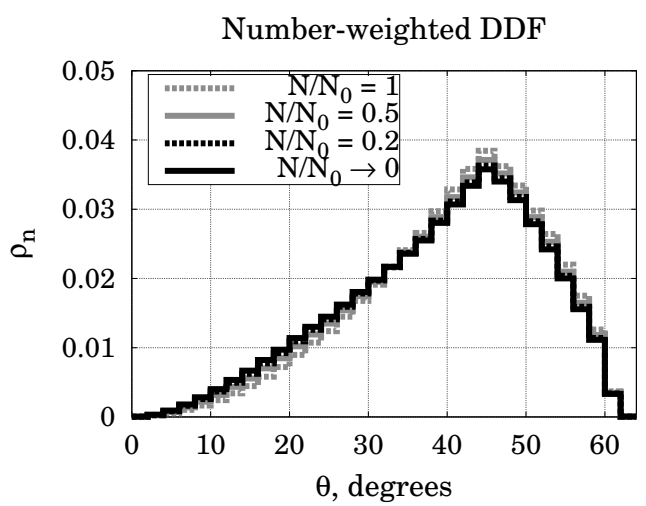

Length-weighted DDF, model $\mathrm{L}_{1}$

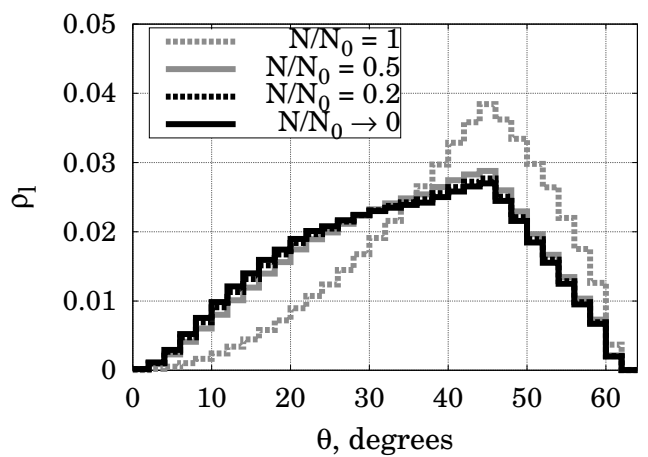

Length-weighted DDF, model $\mathrm{L}_{2}$

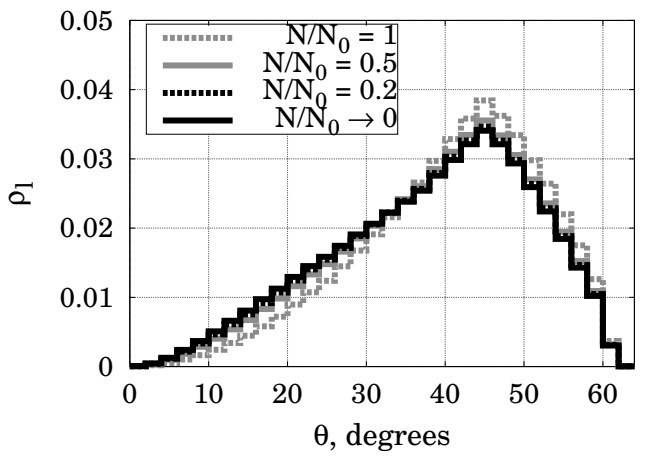

Length-weighted DDF, model $\mathrm{L}_{3}$

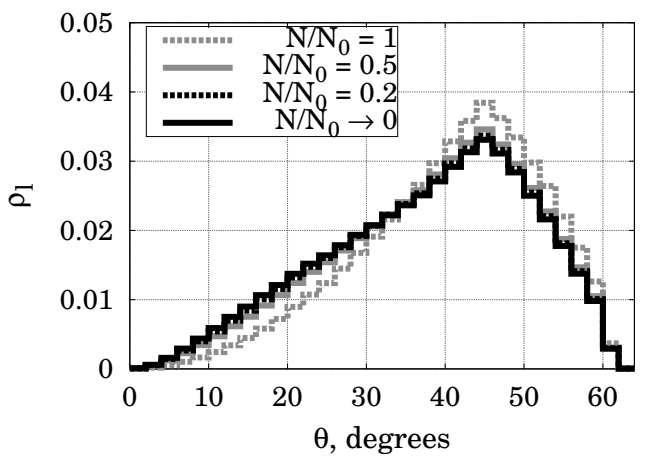

Figure 20: Normalized histograms approximating the number- and lengthweighted DDFs for the grain boundary energy density of Type IV and $\lambda(N) \equiv$ 1.34. Three different boundary lengthening models $\mathbf{L}_{\mathbf{1}}, \mathbf{L}_{\mathbf{2}}, \mathbf{L}_{\mathbf{3}}$ for representing the length-weighted DDF in terms of the number-weighted DDF are implemented. 


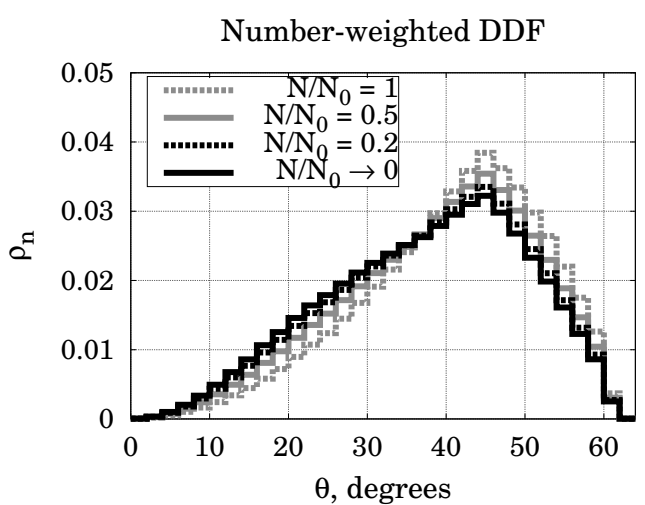

Length-weighted DDF, model $\mathrm{L}_{1}$

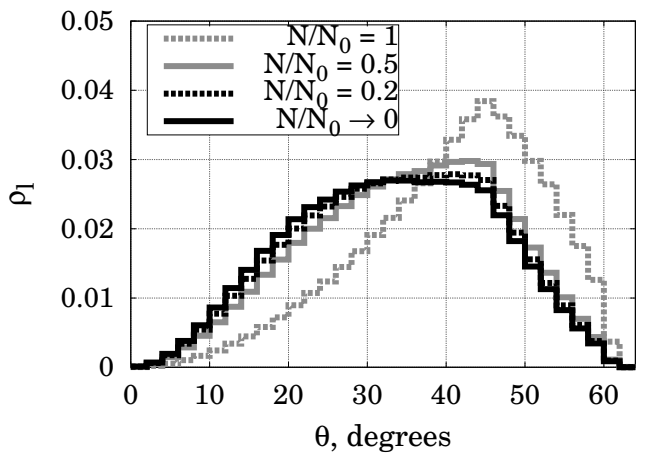

Length-weighted DDF, model $\mathrm{L}_{2}$

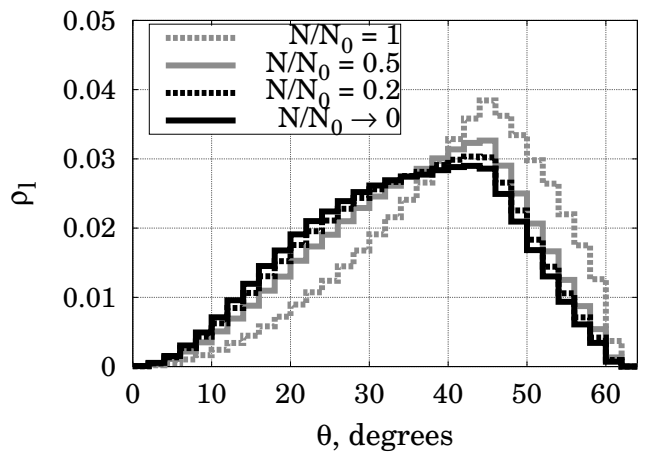

Length-weighted DDF, model $\mathrm{L}_{3}$

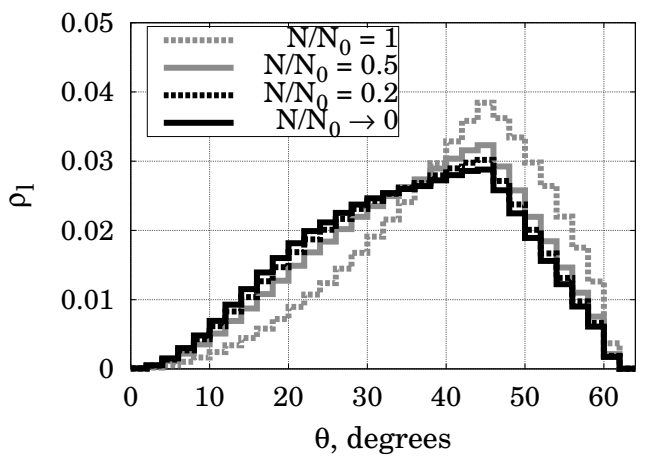

Figure 21: Normalized histograms approximating the number- and lengthweighted DDFs for the grain boundary energy density of Type $\mathbf{V}$ and $\lambda(N) \equiv$ 1.34. Three different boundary lengthening models $\mathbf{L}_{\mathbf{1}}, \mathbf{L}_{\mathbf{2}}, \mathbf{L}_{\mathbf{3}}$ for representing the length-weighted DDF in terms of the number-weighted DDF are implemented.

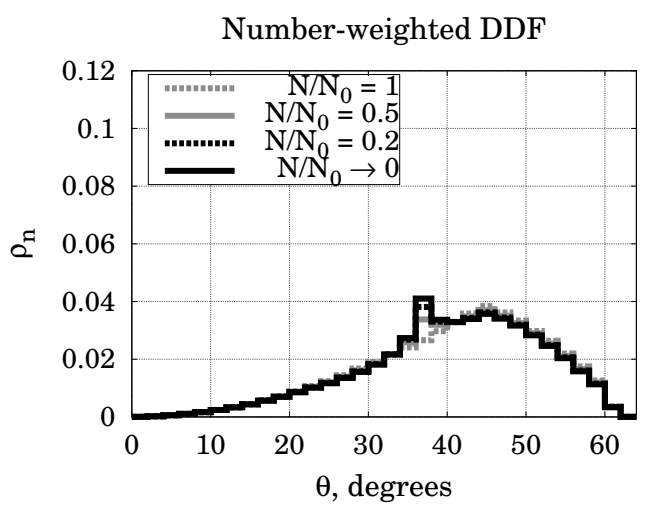

Length-weighted DDF, model $\mathrm{L}_{1}$

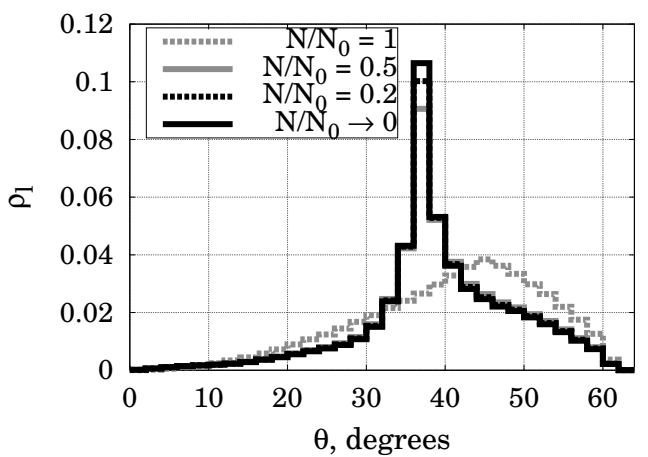

Length-weighted DDF, model $\mathrm{L}_{2}$

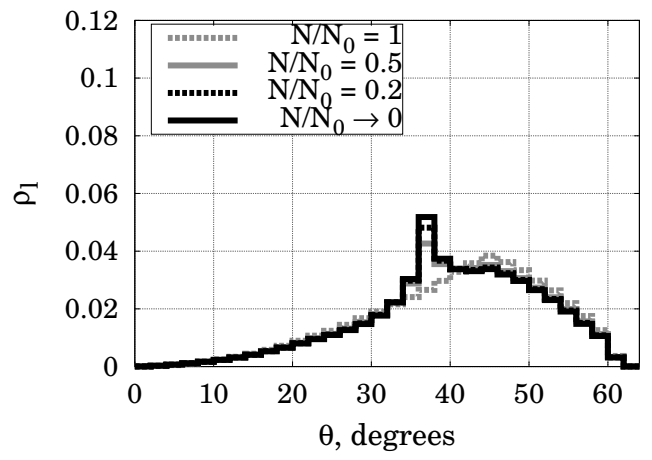

Length-weighted DDF, model $\mathrm{L}_{3}$

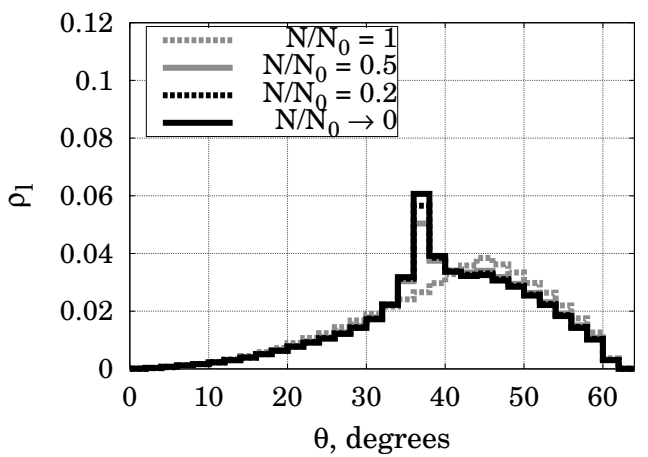

Figure 22: Normalized histograms approximating the number- and lengthweighted DDFs for the grain boundary energy density of Type $\mathbf{V I}$ and $\lambda(N) \equiv$ 1.34. Three different boundary lengthening models $\mathbf{L}_{\mathbf{1}}, \mathbf{L}_{\mathbf{2}}, \mathbf{L}_{\mathbf{3}}$ for representing the length-weighted DDF in terms of the number-weighted DDF are implemented. 
Model $\mathrm{L}_{1}$

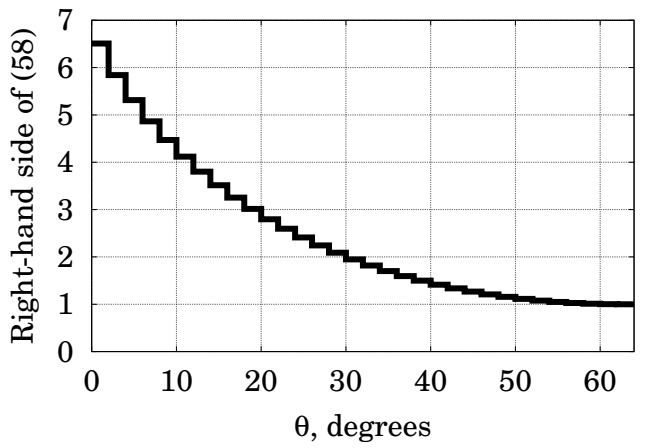

Model $\mathrm{L}_{2}$

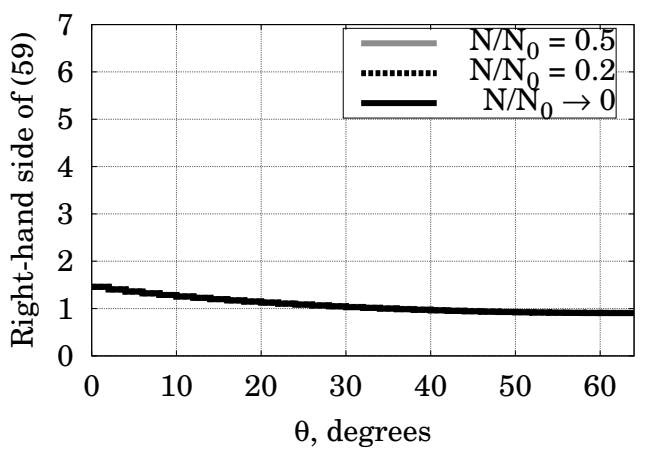

Model $\mathrm{L}_{3}$

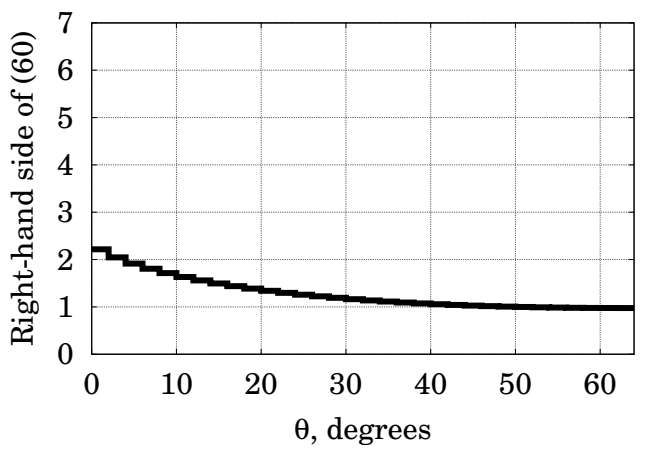

Figure 23: Right-hand sides of (57)-(59) (specifying boundary lengthening models $\left.\mathbf{L}_{\mathbf{1}}, \mathbf{L}_{2}, \mathbf{L}_{3}\right)$ for the grain boundary energy density of Type $\mathbf{I V}$. The righthand side of (58) is computed for the case when $\lambda=\lambda(N)$ is determined by (56) with $\lambda_{\text {isotr }}=1.34, v(x) \equiv x$.
Model $\mathrm{L}_{1}$

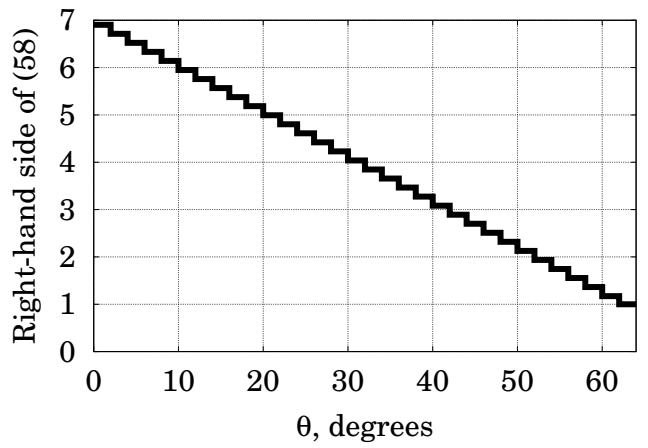

Model $\mathrm{L}_{2}$

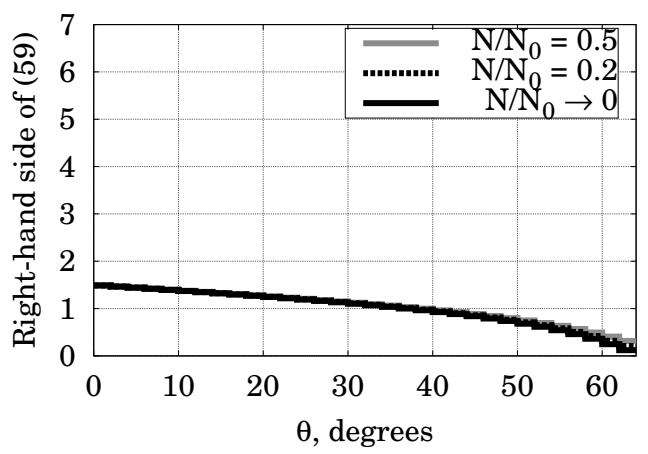

Model $\mathrm{L}_{3}$

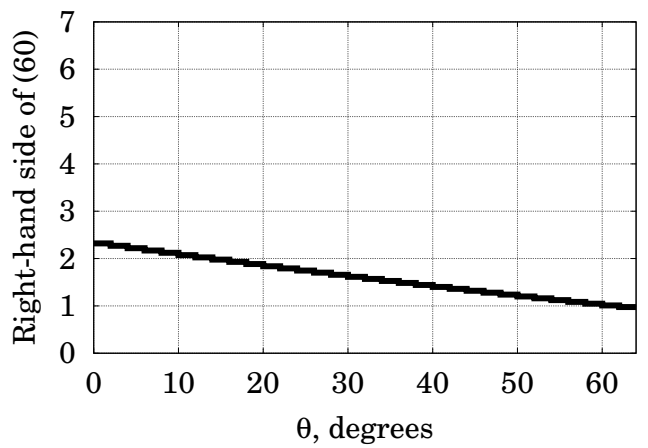

Figure 24: Right-hand sides of (57)-(59) (specifying boundary lengthening models $\left.\mathbf{L}_{\mathbf{1}}, \mathbf{L}_{\mathbf{2}}, \mathbf{L}_{\mathbf{3}}\right)$ for the grain boundary energy density of Type $\mathbf{V}$. The righthand side of (58) is computed for the case when $\lambda=\lambda(N)$ is determined by (56) with $\lambda_{\text {isotr }}=1.34, v(x) \equiv x$. 
Model $\mathrm{L}_{1}$

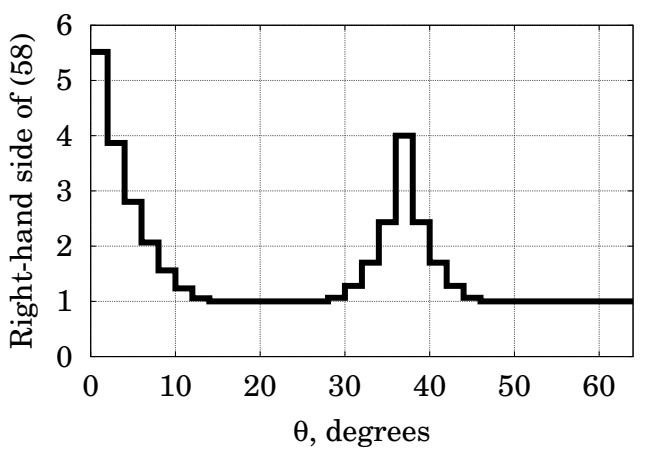

Model $\mathrm{L}_{2}$

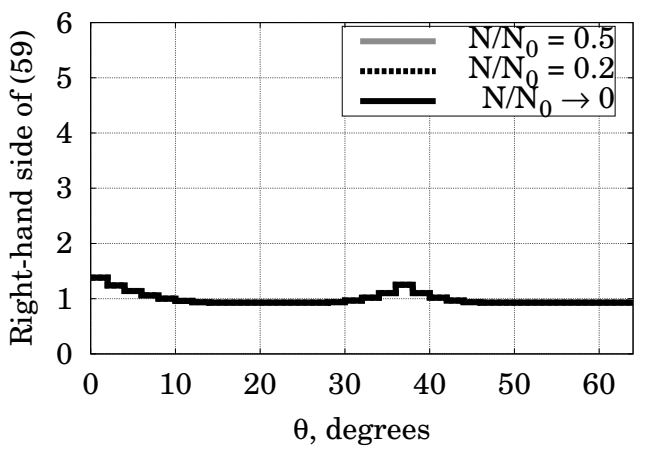

Model $\mathrm{L}_{3}$

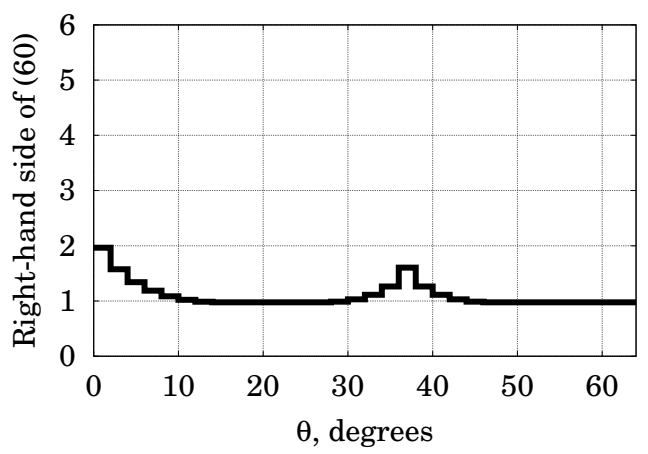

Figure 25: Right-hand sides of (57)-(59) (specifying boundary lengthening models $\left.\mathbf{L}_{\mathbf{1}}, \mathbf{L}_{\mathbf{2}}, \mathbf{L}_{\mathbf{3}}\right)$ for the grain boundary energy density of Type $\mathbf{V I}$. The righthand side of (58) is computed for the case when $\lambda=\lambda(N)$ is determined by (56) with $\lambda_{\text {isotr }}=1.34, v(x) \equiv x$.
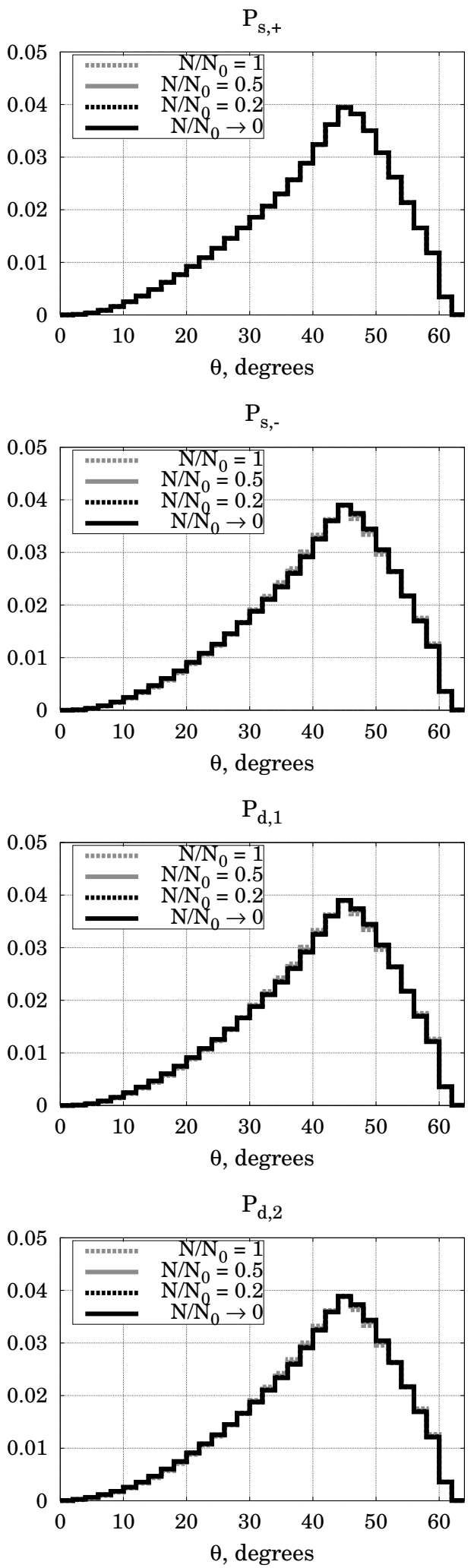

Figure 26: Normalized histograms corresponding to $\left\{P_{s,+}^{i}(N)\right\}_{i=1}^{r}$, $\left\{P_{s,-}^{i}(N)\right\}_{i=1}^{r}, \quad\left\{P_{d, 1}^{i}(N)\right\}_{i=1}^{r}, \quad\left\{P_{d, 2}^{i}(N)\right\}_{i=1}^{r}$ for the grain boundary energy density of Type $\mathbf{I I}$ and $\lambda=\lambda(N)$ determined by (56) with $\lambda_{\mathrm{isotr}}=1.34, v(x) \equiv x$. 


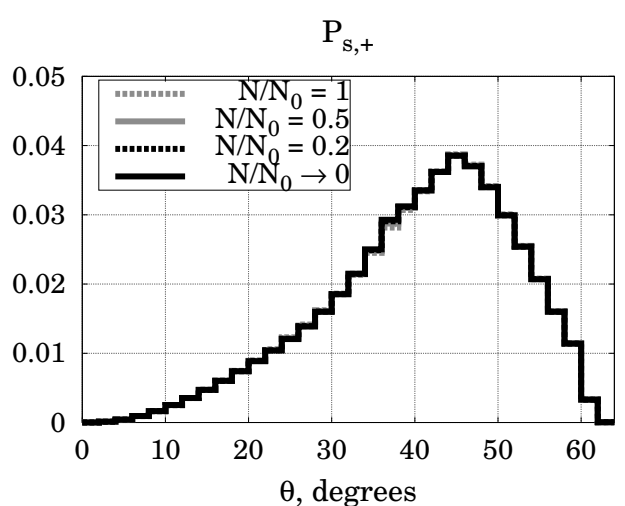

$\mathrm{P}_{\mathrm{s},-}$

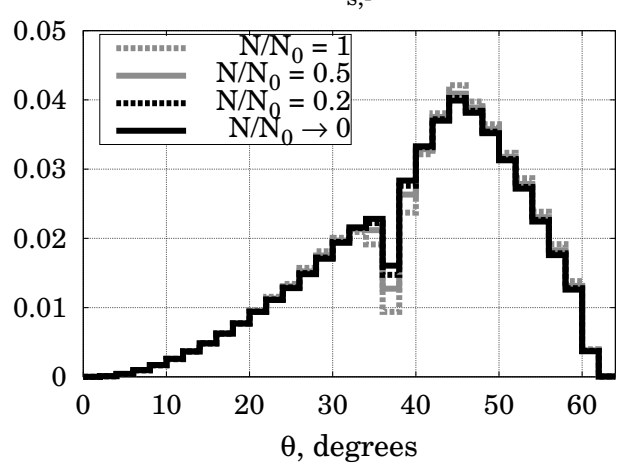

$\mathrm{P}_{\mathrm{d}, 1}$
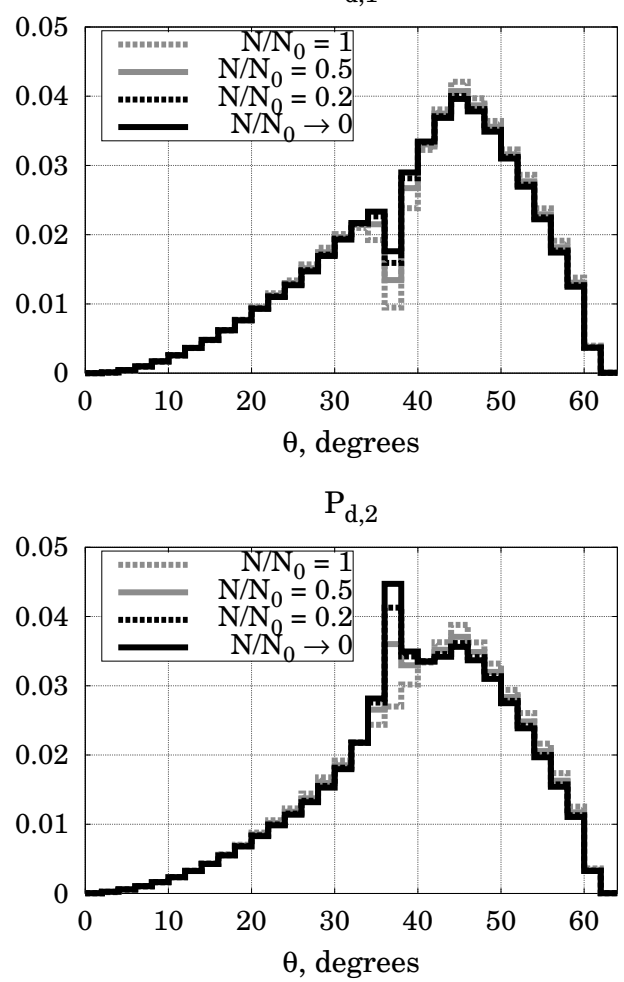

Figure 27: Normalized histograms corresponding to $\left\{P_{s,+}^{i}(N)\right\}_{i=1}^{r}$, $\left\{P_{s,-}^{i}(N)\right\}_{i=1}^{r},\left\{P_{d, 1}^{i}(N)\right\}_{i=1}^{r}, \quad\left\{P_{d, 2}^{i}(N)\right\}_{i=1}^{r}$ for the grain boundary energy density of Type VI and $\lambda=\lambda(N)$ determined by (56) with $\lambda_{\text {isotr }}=1.34$, $v(x) \equiv x$.

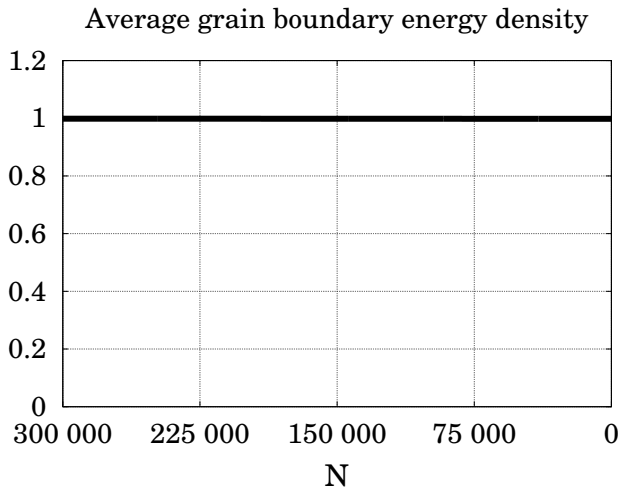

Low- and high-energy boundaries' fractions

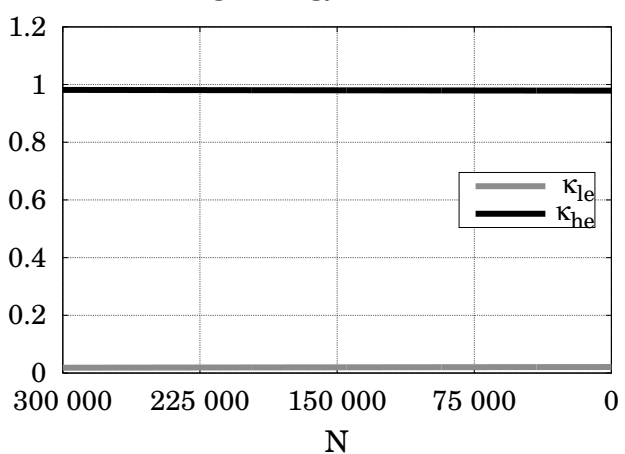

Ratio $\lambda=\lambda(\mathrm{N})$

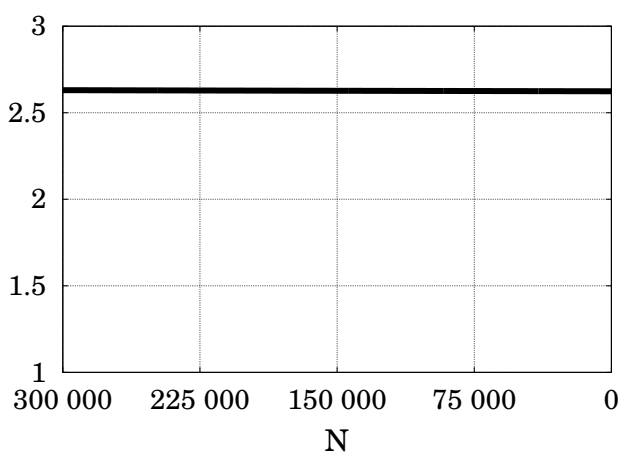

Figure 28: Evolution of the quantities (54),(56) for the grain boundary energy density of Type II. $\operatorname{In}(56), \lambda_{\text {isotr }}=1.34$ and $v(x) \equiv x$. 
Average grain boundary energy density

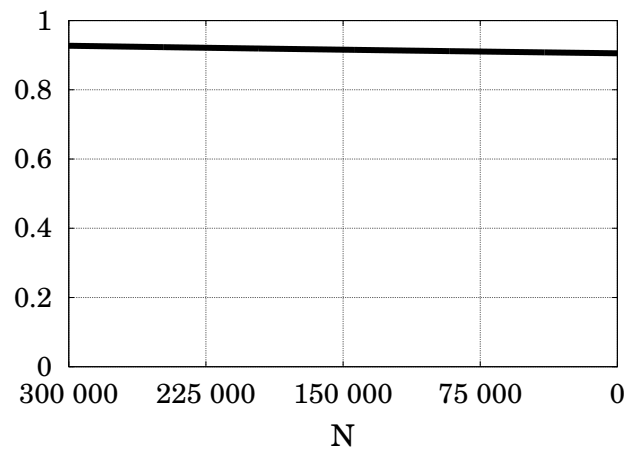

Low- and high-energy boundaries' fractions

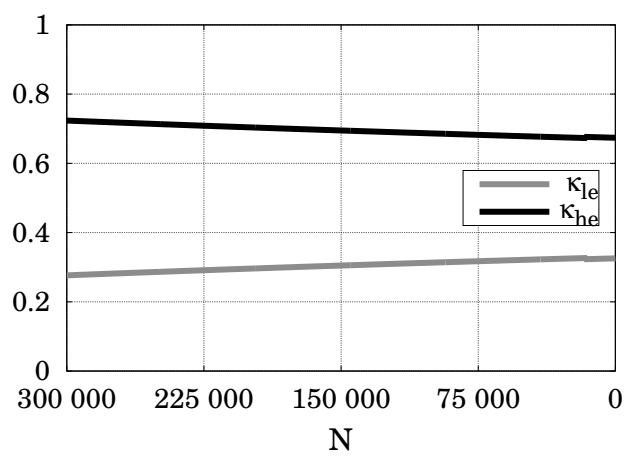

Ratio $\lambda=\lambda(\mathrm{N})$

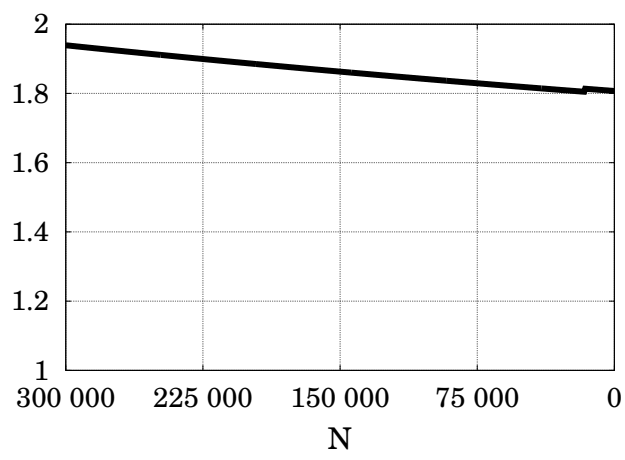

Figure 29: Evolution of the quantities (54),(56) for the grain boundary energy density of Type VI. In (56), $\lambda_{\text {isotr }}=1.34$ and $v(x) \equiv x$. local and global energy minima; see [1, 18]. Thus, the geometric restrictions related to the general-texture case and specified by the a priori distributions $\left\{\eta_{i_{1}, i_{2}}^{i}\right\}_{i=1}^{r}, i_{1}=\overline{1, r}, i_{2}=\overline{1, r}$ (see Figure 6), do not allow grain boundaries with low disorientation angles to be created in large amounts despite the energy reduction principles. In compliance with [14, subsection 2.2] and [13, subsection 4.4], the histograms for $\left\{P_{s,+}^{i}(N)\right\}_{i=1}^{r}$ in Figures 26,27 stay very close to the so-called texture-weighted distribution which is just Mackenzie distribution for general random orientation textures with the cubic lattice symmetry.

3. The boundary lengthening phenomenon makes the lengthweighted DDF evolve stronger than the number-weighted DDF for anisotropic boundary energy densities. The isotropic case is trivial: according to Figure 13, $\rho_{l}=\rho_{n}$ does not evolve. As for the Read-Shockley boundary energy densities of Types II-IV, the corresponding Figures 14-16,19,20 demonstrate that, the greater the cutoff angle, the more the number- and especially length-weighted DDFs increase at low-energy disorientations and, consequently, decrease at high-energy disorientations. By comparing Figures 15,16,19,20 with [13, Figure 5(a)], [14, Figure 7], [23, Figure 4(b)], we deduce the following:

- our kinetic approach together with boundary lengthening model $\mathbf{L}_{\mathbf{2}}$ or $\mathbf{L}_{\mathbf{3}}$ gives results similar to the corresponding results of numerical simulations via Monte Carlo and level set techniques as well as via the kinetic model proposed in [14];

- within the limits of our framework, model $\mathbf{L}_{\mathbf{1}}$ predicts much greater boundary lengthening than obtained from the mentioned large-scale simulations.

Besides, by the use of models $\mathbf{L}_{\mathbf{2}}$ and $\mathbf{L}_{\mathbf{3}}$, we detect an essential qualitative difference between the DDFs for the boundary energy densities of Types IV and $\mathbf{V}$. This difference was illustrated and discussed in [23, subsection 4.1.2, Figure 4]. Indeed, comparing Figures 16,20 with Figures 17,21, respectively, also indicates that, for the affine boundary energy density of Type $\mathbf{V}$ (having the same cutoff disorientation angle $\theta_{\max }$ as the ReadShockley boundary energy density of Type IV), the numberand especially length-weighted DDFs evolve much faster and reflect a considerably stronger preference for low-energy interfaces. As opposed to models $\mathbf{L}_{\mathbf{2}}$ and $\mathbf{L}_{\mathbf{3}}$, model $\mathbf{L}_{\mathbf{1}}$ shows a significant increase in the length fractions of low-energy interfaces for both of the energy density types (and also for Type III as seen from Figure 15). Hence, within the limits of our approach, models $\mathbf{L}_{2}$ and $\mathbf{L}_{3}$ turn out to produce more adequate results than model $\mathbf{L}_{\mathbf{1}}$. Furthermore, among all the considered energy density types, new peaks of the number- and length-weighted DDFs appear only for Type VI at the intermediate local minimum $\theta=\theta_{\mathrm{VI}}$; see Figures 18,22. Unlike the fiber-texture case investigated in $[1,18]$, peaks at $\theta=0$ do not form.

4. Figures 28,29 illustrate the following property. Suppose that the Read-Shockley boundary energy density of Type II is replaced with its modification in the form of the boundary energy density of Type VI (having an additional local minimum at $\left.\theta=\theta_{\mathrm{VI}}>\theta_{\mathrm{II}}\right)$. Then the average energy density $\bar{\gamma}=\bar{\gamma}(N)$ and fraction $\kappa_{\mathrm{he}}=\kappa_{\mathrm{he}}(N)$ of high-energy boundaries take smaller 
values and decrease a little bit faster with decreasing $N$, while the fraction $\kappa_{\mathrm{le}}=\kappa_{\mathrm{le}}(N)$ of low-energy boundaries takes greater values and increases a little bit faster with decreasing $N$.

\section{Concluding remarks}

In this paper, a Boltzmann-type kinetic modeling approach for two-dimensional polycrystalline grain growth was proposed. This was an extension of the fiber-texture framework developed in [1] to the case of general textures with anisotropic grain boundary energy densities depending only on the disorientation angle. The number-weighted disorientation distribution function was approximated numerically by solving a specific system of ordinary differential equations, where the time variable was replaced with the time-decreasing total number of grain boundaries. These equations were initially derived in a discretized form with respect to the disorientation variable. Besides, in order to obtain their terms describing the influence of topological transitions on the number-weighted disorientation distribution function, a collection of auxiliary a priori distributions was used. The latter concerned possible disorientations of grain boundaries connected in a triple junction and were numerically constructed for the technologically important subcase of the cubic lattice symmetry taking place in a wide class of metals and alloys. As in $[1,14]$, the ratio of the length- and number-weighted disorientation distribution functions was estimated via boundary lengthening models. Three variants of such models were compared with each other in terms of the obtained numerical results.

Unlike the evolving number- and length-weighted disorientation distributions, the mentioned a priori distributions have to be constructed only once for the cubic or any other particular lattice symmetry and depend on none of such quantities as the grain boundary energy density, ratio of the neighbor switchings' rate to the grain disappearances' rate, initial number of grain boundaries, initial number- and length-weighted disorientation distributions.

The numerical results obtained via the presented approach showed good agreement with the corresponding large-scale simulation results from $[14,23]$.

Moreover, the developed framework is based on solving a system of kinetic ordinary differential equations and, therefore, a priori more computationally efficient than large-scale simulation methods.

As a future perspective, it is worth investigating to extend the proposed general-texture approach by introducing the intragranular stored energy variable into the kinetic model, which may help to investigate primary recrystallization as well as grain growth. Then, in addition to neighbor switchings and grain disappearances, one new type of topological transitions has to be taken into account. It arises only in the presence of differences between grains' stored energies, when a vertex, i. e., a junction of grain boundaries, collides with the interior of some other boundary; see [33-35].

\section{Acknowledgements}

I. Y. was supported in part by National Science Foundation grant DMS-1056821 and Russian state subsidy for applied scientific research 14.607.21.0091. M. E. was supported in part by National Science Foundation grant DMS-1056821.

\section{References}

1. Yegorov, I., Torres, C.E., Emelianenko, M. A Boltzmann-type kinetic model for misorientation distribution functions in two-dimensional fibertexture polycrystalline grain growth. Acta Materialia 2016; 109:230-247.

2. Randle, V., Owen, G. Mechanisms of grain boundary engineering. Acta Materialia 2006; 54:1777-1783.

3. Krupp, U., Kane, W.M., Liu, X., Dueber, O., Laird, C., McMahon Jr., C. J. The effect of grain-boundary-engineering-type processing on oxygen-induced cracking of IN718. Materials Science and Engineering A $2003 ; 349: 213-217$.

4. Alexandreanu, B., Was, G. S. The role of stress in the efficacy of coincident site lattice boundaries in improving creep and stress corrosion cracking. Scripta Materialia 2006; 54:1047-1052.

5. Gupta, G., Alexandreanu, B., Was, G. S. Grain boundary engineering of ferritic-martensitic alloy T91. Metallurgical and Materials Transactions 2004; 35A:717-719.

6. Furuhara, T., Maki, T. Grain boundary engineering for superplasticity in steels. Journal of Materials Science 2005; 40:919-926.

7. Kurban, M., Erb, U., Aust, K. T. A grain boundary characterization study of boron segregation and carbide precipitation in alloy 304 austenitic stainless steel. Scripta Materialia 2006; 54:1053-1058.

8. Smith, W., Hashemi, J. Foundations of Materials Science and Engineering. McGraw-Hill Education: London, 2009.

9. Gottstein, G., Shvindlerman, L.S. Grain Boundary Migration in Metals: Thermodynamics, Kinetics, Applications. Taylor \& Francis Group: Boca Raton, 2010.

10. Holm, E. A., Hassold, G. N., Miodownik, M. A. On misorientation distribution evolution during anisotropic grain growth. Acta Materialia 2001; 49:2981-2991.

11. Moldovan, D., Wolf, D., Phillpot, S. R., Haslam A. J. Mesoscopic simulation of two-dimensional grain growth with anisotropic grain-boundary properties. Philosophical Magazine A 2002; 82(7):1271-1297.

12. Upmanyu, M., Hassold, G. N., Kazaryan, A., Holm, E. A., Wang, Y., Patton, B., Srolovitz, D. J. Boundary mobility and energy anisotropy effects on microstructural evolution during grain growth. Interface Science 2002; 10(2):201-216.

13. Gruber, J., Miller, H. M., Hoffmann, T.D., Rohrer, G. S., Rollett, A.D. Misorientation texture development during grain growth. Part I: Simulation and experiment. Acta Materialia 2009; 57:6102-6112.

14. Gruber, J., Rohrer, G. S., Rollett, A. D. Misorientation texture development during grain growth. Part II: Theory. Acta Materialia 2010; 58:14-19.

15. Rohrer, G.S., Gruber, J., Rollett, A.D. A model for the origin of anisotropic grain boundary character distributions in polycrystalline materials. In Applications of Texture Analysis, Rollett, A. D. (Editor), Ceramic Transactions, 201. John Wiley \& Sons: Hoboken, New Jersey, 2009.

16. Dillon, S. J., Rohrer, G. S. Mechanism for the development of anisotropic grain boundary character distributions during normal grain growth. Acta Materialia 2009; 57(1):1-7.

17. Kazaryan, A., Wang, Y., Dregia, S. A., Patton, B. R. Grain growth in anisotropic systems: comparison of effects of energy and mobility. Acta Materialia 2002; 50:2491-2502.

18. Moelans, N., Spaepen, F., Wollants P. Grain growth in thin films with a fiber texture studied by phase-field simulations and mean field modeling. Philosophical Magazine 2010; 90(1-4):501-523.

19. Kinderlehrer, D., Lee, J., Livshits, I., Rollett, A., Ta'asan, S. Mesoscale simulation of grain growth. Materials Science Forum 2004; 467-470:10571062.

20. Kinderlehrer, D., Livshits, I., Rohrer, G. S., Ta'asan, S., Yu, P. Mesoscale simulation of the evolution of the grain boundary character distribution. $M a$ terials Science Forum 2004; 467-470:1063-1068. 
1 21. Kinderlehrer, D., Livshits, I., Ta'asan, S. A variational approach to modeling and simulation of grain growth. SIAM Journal on Scientific Computing 2006; 28(5):1694-1715.

3 22. Barmak, K., Emelianenko, M., Golovaty, D., Kinderlehrer, D., Ta'asan, S. A new perspective on texture evolution. International Journal of Numerical Analysis and Modeling 2008; 5:93-108.

6 23. Elsey, M., Esedoglu, S., Smereka, P. Simulations of anisotropic grain growth: Efficient algorithms and misorientation distributions. Acta Materialia 2013; 61(6):2033-2043.

8 24. Hallberg, H. Influence of anisotropic grain boundary properties on the evo-

9 lution of grain boundary character distribution during grain growth - A 2D

10 level set study. Modelling and Simulation in Materials Science and Engineering 2014; 22(8), 085005.

12 25. Chang, K., Moelans, N. Effect of grain boundary energy anisotropy on highly-textured grain structures studied by phase field simulations. Acta Materialia 2014; 64:443-454.

14 26. Weygand, D., Brechet, Y., Lepinoux, J. A vertex dynamics simulation of 15 grain growth in two dimensions. Philosophical Magazine B 1998; 78(4):32916352

17 27. Henseler, R., Niethammer, B., Otto, F. A reduced model for simulating grain growth. Free Boundary Problems 2004; Volume 147 of the series ISNM International Series of Numerical Mathematics:177-187.

19 28. Torres, C. E., Emelianenko, M., Golovaty, D., Kinderlehrer, D., Ta'asan, S.

20 Numerical analysis of the vertex models for simulating grain boundary networks. SIAM Journal on Applied Mathematics 2015; 75(2):762-786.

. Engler, O., Randle, V. Introduction to Texture Analysis: Macrotexture, $M i$ crotexture, and Orientation Mapping. Taylor and Francis Group: Boca Raton, 2010.

4 30. Grimmer, H. Disorientations and coincidence rotations for cubic lattices.

25 Acta Crystallographica 1974; A30:685-688.

26 31. Heinz, A., Neumann, P. Representation of orientation and disorientation data for cubic, hexagonal, tetragonal and orthorhombic crystals. Acta Crystallographica 1991; A47:780-789.

28 32. Sprague, A. P., Patterson, B. R., Grandhi, S. Topological characteristics of 29 two-dimensional grain growth - Simulation and analysis. Metallurgical and 30 Materials Transactions A 2010; 41(3):592-602.

31 33. Piekos, K., Tarasiuk, J., Wierzbanowski, K., Bacroix, B. Recrystallization study using two-dimensional vertex model. Archives of Metallurgy and Materials 2005; 50(1):131-138.

33 34. Piekos, K., Tarasiuk, J., Wierzbanowski, K., Bacroix, B. Stochastic vertex model of recrystallization. Computational Materials Science 2008; 42:3642.

36 35. Piekos, K., Tarasiuk, J., Wierzbanowski, K., Bacroix, B. Generalized ver37 tex model of recrystallization - Application to polycrystalline copper. Com38 putational Materials Science 2008; 42:584-594. 


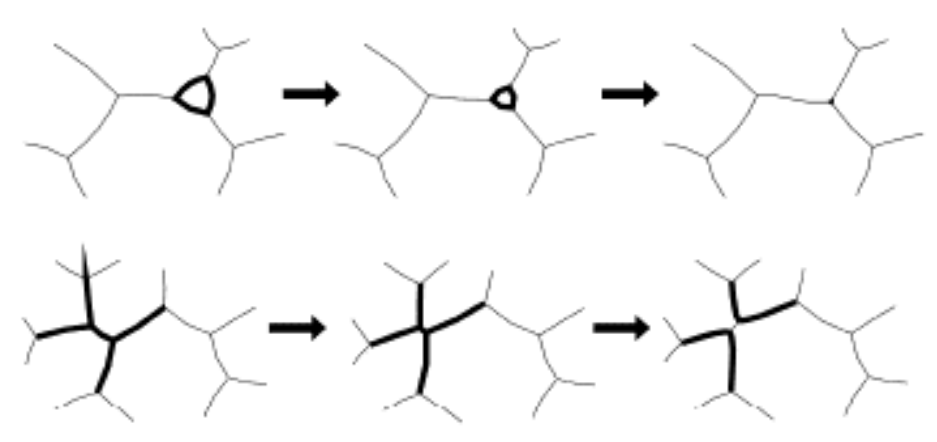

Geometric restrictions on the disorientations of grain boundaries in general-texture networks
Fundamental laws governing 2D polyctrystalline grain growth with the corresponding topological transitions

Boltzmann-type kinetic model (in a specific discretized form with respect to the disorientation variable) based on a system of ODEs

Approximation of the number-weighted DDF
The developed framework is tested by comparing the numerical results, which it gives for several benchmark examples, with the corresponding large-scale simulation results obtained by Monte Carlo and level set methods.

Three different boundary lengthening models are compared with each other.

The proposed approach is a priori less computationally expensive than large-scale direct simulation techniques.

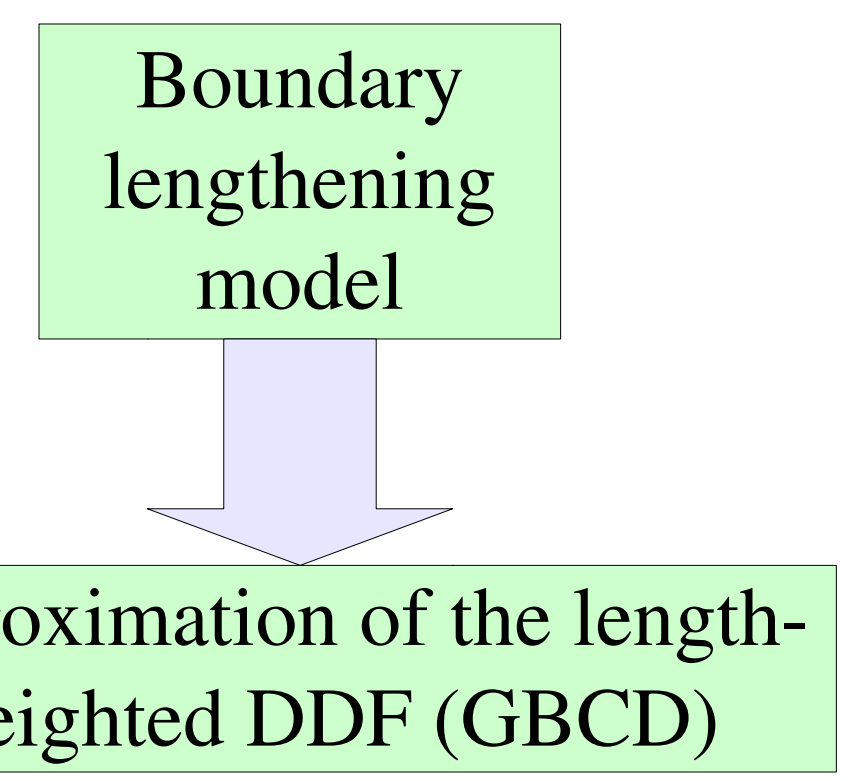

